\title{
THE SURVIVAL PROBABILITY OF A CRITICAL MULTI-TYPE BRANCHING PROCESS IN I.I.D. RANDOM ENVIRONMENT
}

\author{
By E. Le PAGE*, M. PeIGné ${ }^{\dagger}$ And C. Pham ${ }^{\dagger}$ \\ Université de Bretagne-Sud * and Université Fr. Rabelais Tours ${ }^{\dagger}$ \\ We study the asymptotic behaviour of the probability of non-extinction \\ of a critical multi-type Galton-Watson process in i.i.d. random environments \\ by using limit theorems for products of positive random matrices. Under suit- \\ able assumptions, the survival probability is proportional to $1 / \sqrt{n}$.
}

1. Introduction. Branching processes in random environments (BPREs) were introduced in the 1960s (see, for instance, [22]) to describe the development of populations whose evolution may be affected by environmental factors; they have been a central topic of research.

In the single-type case, the behaviour of these processes is mainly determined by the 1-dimensional random walk generated by the logarithms of the expected population sizes $m_{k}, k \geq 0$, of the respective generations. The theory of fluctuations of random walks on $\mathbb{R}$ with i.i.d. increments allows one to classify BPREs in three classes - supercritical, critical or subcritical-of single-type BPREs, according to the fact that the associated random walk tends to $+\infty$, oscillates or tends to $-\infty$ (see, for instance, the fundamental papers $[2,3])$. In particular, when $\mathbb{E}\left(\left|\log m_{k}\right|\right)<+\infty$, the BPRE is supercritical (resp., critical or subcritical) when $\mathbb{E}\left(\log m_{k}\right)>0$ [resp., $\mathbb{E}\left(\log m_{k}\right)=0$ or $\left.\mathbb{E}\left(\log m_{k}\right)<0\right]$. There exist numerous statements concerning the asymptotic behaviour of the probability of nonextinction up to time $n$, the distribution of the population size conditionally to survival up to moment $n$, large deviation type results (see, for instance, $[1,4,7]$ ). In the critical case, the branching process becomes extinct with probability one [2]; M. V. Kozlov [19] (for BPREs with linearly fractional generating functions) and J. Geiger and G. Kersting [10] in the general case strengthened this result and proved that the probability of nonextinction up to time $n$ is equivalent to $c_{1} / \sqrt{n}$ as $n \rightarrow+\infty$, for some explicit constant $c_{1}>0$ (see also [15]). Let us recall that this probability of nonextinction up to time $n$ is equivalent to $1 / n$ when the offspring distribution is fixed, that is, it does not vary randomly; in other words, branching processes in a random environment die more slowly. In the supercritical and subcritical cases, similar studies have been done (see, for instance, $[2,3,11,16]$ ); we do not go into detail about these cases since they are outside the scope of this paper.

Received October 2016; revised November 2017.

MSC2010 subject classifications. Primary 60J80; secondary 60F17, 60K37.

Key words and phrases. Multi-type branching process, survival probability, random environment, product of random matrices, critical case. 
It is of interest to prove analogues of the above statements for the multi-type BPREs $\left(Z_{n}\right)_{n \geq 0}$. The main difficulty which appears is that the role of the random walk associated to the BPRE in this case is played by the logarithm of the norm of some $\mathbb{R}^{p}$-valued Markov chain whose increments are governed by i.i.d. random $p \times p$-matrices $M_{k}, k \geq 0$; the coefficients of these matrices $M_{k}$ are nonnegative and correspond to the expected population sizes of the respective generations, according to the types of the particles and their direct parents. Products of random matrices have been the object of many investigations and many limit theorems do exist in this context: for instance, the law of large numbers, the central limit theorem and the large deviations principle (see $[5,20]$ and references therein). Unfortunately, the theory of their fluctuations remains a field in which practically no research has been done and the multi-type BPREs constitute a relevant application area for this study. Nevertheless, as in the single-type case, the set of multi-type BPREs may be divided into three classes: they are supercritical (resp., critical or subcritical) when the upper Lyapunov exponent of the associated random matrices is positive, null or negative [18].

A problem of particular importance is to specify the asymptotic behaviour of their probability of nonextinction up to time $n$. As for single-type BPREs, the case has been first studied when the generating functions defining the random environment are linear fractional; it yields to explicit formulas which are easier to tackle.

In [6], E. E. Dyakonova obtained in the critical case an equivalence of the survival probability at time $n$, under the restrictive assumption that the mean matrices $M_{k}$ have a concordant deterministic Perron-Frobenius eigenvector. It happens, for instance, when the $M_{k}$ are upper-triangular matrices with strictly decreasing coefficients on the diagonal. In this case, the behaviour of the BPRE is governed by the 1-dimensional random walk whose increments are the logarithm of the spectral radius of the $M_{k}$; this allows one to apply the same techniques as in the single-type case.

To tackle the "general" case when the action of the random matrices $M_{k}$ is strongly irreducible (see hypothesis $\mathrm{H} 2$ below), limit theorems on the fluctuations of the norm of products of random matrices were required; they have been recently achieved in [12] and [13] with asymptotic results on the tails of certain hitting time distribution.

In this paper, we investigate the asymptotic behaviour of the probability of nonextinction up to time $n$ of critical multi-type BPREs and obtain an optimal result in the case of linear fractional generating functions. To formulate our main results, we first introduce some standard notation and definitions.

We fix an integer $p \geq 2$ and denote $\mathbb{R}^{p}$ (resp., $\mathbb{N}^{p}$ ) the set of $p$-dimensional column vectors with real (resp., nonnegative integers) coordinates; for any column vector $x=\left(x_{i}\right)_{1 \leq i \leq p} \in \mathbb{R}^{p}$, we denote $\tilde{x}$ the row vector $\tilde{x}:=\left(x_{1}, \ldots, x_{p}\right)$. Let $\mathbf{1}$ (resp., 0) be the column vector of $\mathbb{R}^{p}$ whose all coordinates equal 1 (resp., 0 ). We fix a basis $\left\{e_{i}, 1 \leq i \leq p\right\}$ in $\mathbb{R}^{p}$ and denote $|\cdot|$ the corresponding $L^{1}$ norm. We 
also consider the general linear semi-group $S^{+}$of $p \times p$ matrices with positive coefficients. We endow $S^{+}$with the $L^{1}$-norm denoted also by $|\cdot|$.

The multi-type Galton-Watson process is a temporally homogeneous vector Markov process $\left(Z_{n}\right)_{n \geq 0}$ whose states are row vectors in $\mathbb{N}^{p}$. We always assume that $Z_{0}$ is nonrandom. For any $1 \leq i \leq p$, the $i$ th component $Z_{n}(i)$ of $Z_{n}$ may be interpreted as the number of objects of type $i$ in the $n$th generation.

We consider a measurable function $\xi \mapsto f_{\xi}$ from $\mathbb{R}$ to the set of multivariate probability generating functions $f_{\xi}=\left(f_{\xi}^{(i)}\right)_{1 \leq i \leq p}$ defined by

$$
f_{\xi}^{(i)}(s)=\sum_{\alpha \in \mathbb{N} p} p_{\xi}^{(i)}(\alpha) s^{\alpha},
$$

for any $s=\left(s_{i}\right)_{1 \leq i \leq p} \in[0,1]^{p}$, where:

(i) $\alpha=\left(\alpha_{i}\right)_{i} \in \mathbb{N}^{p}$ and $s^{\alpha}=s_{1}^{\alpha_{1}} \ldots s_{p}^{\alpha_{p}}$;

(ii) $p_{\xi}^{(i)}(\alpha)=p_{\xi}^{(i)}\left(\alpha_{1}, \ldots, \alpha_{p}\right)$ is the probability that an object of type $i$ in environment $\xi$ has $\alpha_{1}$ children of type $1, \ldots, \alpha_{p}$ children of type $p$.

Let $\left(\xi_{n}\right)_{n \geq 0}$ be a sequence of real valued i.i.d. random variables defined on a probability space $(\Omega, \mathcal{F}, \mathbb{P})$. The Galton-Watson process with $p$ types of particles in a random environment $\left(\xi_{n}\right)_{n \geq 0}$ describes the evolution of a particle population $Z_{n}=\left(Z_{n}(i)\right)_{1 \leq i \leq p}$ for $n \geq 0$.

We assume that for any $n \geq 0, i=1, \ldots, p$ and $\xi \in \mathbb{R}$, if $\xi_{n}=\xi$ then each of the $Z_{n}(i)$ particles of type $i$ existing at time $n$ produces offspring in accordance with the $p$-dimensional generating function $f_{\xi}^{(i)}(s)$ independently of the reproduction of other particles of all types.

If $Z_{0}=\tilde{e}_{i}$, then $Z_{1}$ has the generating function

$$
f_{\xi_{0}}^{(i)}(s)=\sum_{\alpha \in \mathbb{N} p}^{+\infty} p_{\xi_{0}}^{(i)}(\alpha) s^{\alpha} .
$$

In general, if $Z_{n}=\left(\alpha_{i}\right)_{1 \leq i \leq p}$, then $Z_{n+1}$ is the sum of $\alpha_{1}+\cdots+\alpha_{p}$ independent random vectors, where $\alpha_{i}$ particles of type $i$ have the generating function $f_{\xi_{n}}^{(i)}$ for $i=1, \ldots, p$. It is obvious that if $Z_{n}=\tilde{\mathbf{0}}$, then $Z_{n+1}=\tilde{\mathbf{0}}$.

Denote $f_{n}=f_{\xi_{n}}$. By the above descriptions (written in equation (2.1) in [18]), for any $s=\left(s_{i}\right)_{1 \leq i \leq p}, 0 \leq s_{i} \leq 1$,

$$
\mathbb{E}\left(s^{Z_{n}} \mid Z_{0}, \ldots, Z_{n-1}, f_{0}, \ldots, f_{n-1}\right)=f_{n-1}(s)^{Z_{n-1}}
$$

which yields (Lemma 2.1 in [18])

$$
\begin{aligned}
\mathbb{E}\left(s^{Z_{n}} \mid f_{0}^{(i)}, f_{1}, \ldots, f_{n-1}\right) & :=\mathbb{E}\left(s^{Z_{n}} \mid Z_{0}=\tilde{e}_{i}, f_{0}, \ldots, f_{n-1}\right) \\
& =f_{0}^{(i)}\left(f_{1}\left(\ldots f_{n-1}(s) \ldots\right)\right) .
\end{aligned}
$$


In particular, the probability of nonextinction $q_{n}^{(i)}$ at generation $n$ given the environment $f_{0}^{(i)}, f_{1}, \ldots f_{n-1}$ is

$$
\begin{aligned}
q_{n}^{(i)} & :=\mathbb{P}\left(Z_{n} \neq \tilde{\mathbf{0}} \mid f_{0}^{(i)}, f_{1}, \ldots, f_{n-1}\right) \\
& =1-f_{0}^{(i)}\left(f_{1}\left(\ldots f_{n-1}(\tilde{\mathbf{0}}) \ldots\right)\right)=\tilde{e}_{i}\left(\mathbf{1}-f_{0}\left(f_{1}\left(\ldots f_{n-1}(\tilde{\mathbf{0}}) \ldots\right)\right)\right),
\end{aligned}
$$

so that

$$
\mathbb{E}\left[q_{n}^{(i)}\right]=\mathbb{E}\left[\mathbb{P}\left(Z_{n} \neq \tilde{\mathbf{0}} \mid f_{0}^{(i)}, f_{1}, \ldots, f_{n-1}\right)\right]=\mathbb{P}\left(Z_{n} \neq \tilde{\mathbf{0}} \mid Z_{0}=e_{i}\right) .
$$

As in the classical one-type case, the asymptotic behaviour of the quantity above is controlled by the mean of the offspring distributions. We assume that the offspring distributions have finite first and second moments; the generating functions $f_{\xi}^{(i)}, \xi \in \mathbb{R}, 1 \leq i \leq p$, are thus $C^{2}$-functions on $[0,1]^{p}$ and we introduce:

(i) the random mean matrices $M_{\xi_{n}}=\left(M_{\xi_{n}}(i, j)\right)_{1 \leq i, j \leq p}=\left(\frac{\partial f_{\xi_{n}}^{(i)}(\mathbf{1})}{\partial s_{j}}\right)_{i, j}$ taken from the vector-valued random generating function $f_{\xi_{n}}(s)$ at $s=\mathbf{1}$, namely

$$
M_{\xi_{n}}=\left(\begin{array}{ccc}
\frac{\partial f_{\xi_{n}}^{(1)}(\mathbf{1})}{\partial s_{1}} & \ldots & \frac{\partial f_{\xi_{n}}^{(1)}(\mathbf{1})}{\partial s_{p}} \\
\vdots & & \\
\frac{\partial f_{\xi_{n}}^{(p)}(\mathbf{1})}{\partial s_{1}} & \ldots & \frac{\partial f_{\xi_{n}}^{(p)}(\mathbf{1})}{\partial s_{p}}
\end{array}\right) .
$$

(ii) the random Hessian matrices $B_{\xi_{n}}^{(i)}=\left(B_{\xi_{n}}^{(i)}(k, l)\right)_{1 \leq k, l \leq p}=\left(\frac{\partial^{2} f_{\xi_{n}}^{(i)}}{\partial s_{k} \partial s_{l}}(\mathbf{1})\right)_{k, l}$, $1 \leq i \leq p$, taken from the real-valued random generating function $f_{\xi_{n}}^{(i)}(s)$ at $s=\mathbf{1}$.

The random variables $M_{\xi_{n}}$ and $B_{\xi_{n}}^{(i)}$ are i.i.d. The common law of the $M_{\xi_{n}}$ is denoted by $\mu$ and for the sake of brevity, we write $M_{n}$ instead of $M_{\xi_{n}}$.

Let $R_{n}$ and $L_{n}$ denote the right and the left product of random matrices $M_{k}$, $k \geq 0$, respectively, $R_{n}=M_{0} M_{1} \ldots M_{n-1}$ and $L_{n}=M_{n-1} \ldots M_{1} M_{0}$.

By [9], if $\mathbb{E}\left(\max \left(0, \log \left|M_{0}\right|\right)\right)<+\infty$, then the sequence $\left(\frac{1}{n} \log \left|R_{n}\right|\right)_{n \geq 1}$ converges $\mathbb{P}$-almost surely to some constant limit $\pi_{\mu}:=\lim _{n \rightarrow+\infty} \frac{1}{n} \mathbb{E}\left[\log \left|R_{n}\right|\right]$. Furthermore, by [18], if there exists a constant $A>0$ such that $\frac{1}{A} \leq M_{\xi_{n}}(i, j) \leq A$ and $0 \leq B_{\xi_{n}}^{(i)}(k, l) \leq A \mathbb{P}$-almost surely for any $1 \leq i, j, k, l \leq p$, then the process $\left(Z_{n}\right)_{n \geq 0}$ becomes extinct $\mathbb{P}$-almost surely if and only if $\pi_{\mu} \leq 0$.

In the present work, we focus our attention on the so-called critical case, that is, $\pi_{\mu}=0$, and specify the speed of extinction of the Galton-Watson process.

We define the cone $\mathcal{C}$ and the space $\mathbb{X}$, respectively, as follows:

$$
\mathcal{C}:=\left\{\tilde{x}=\left(x_{1}, \ldots, x_{p}\right) \in \mathbb{R}^{p} \mid \forall i=1, \ldots, p, x_{i} \geq 0\right\}
$$


and

$$
\mathbb{X}:=\{\tilde{x} \in \mathcal{C}|| \tilde{x} \mid=1\} .
$$

In the sequel, we consider:

- the right and the left linear actions of $S^{+}$on $\mathcal{C}$ defined by

$$
(\tilde{x}, g) \mapsto \tilde{x} g \quad \text { and } \quad(\tilde{x}, g) \mapsto g x
$$

for any $\tilde{x} \in \mathcal{C}$ and $g \in S^{+}$,

- the right and the left projective actions of $S^{+}$on $\mathbb{X}$ defined by

$$
(\tilde{x}, g) \mapsto \tilde{x} \cdot g=\frac{\tilde{x} g}{|\tilde{x} g|} \quad \text { and } \quad(\tilde{x}, g) \mapsto g \cdot x=\frac{g x}{|g x|}
$$

for any $\tilde{x} \in \mathbb{X}$ and $g \in S^{+}$.

For any $g=(g(i, j))_{1 \leq i, j \leq d} \in S^{+}$, let $v(g):=\min _{1 \leq j \leq d}\left(\sum_{i=1}^{d} g(i, j)\right)$. Then, for any $x \in \mathcal{C}$,

$$
0<v(g)|x| \leq|g x| \leq|g||x| .
$$

We set $N(g):=\max \left(\frac{1}{v(g)},|g|\right)$. We also introduce some proper subset of $S^{+}$which is of interest in the sequel: for any constant $B \geq 1$, let $S^{+}(B)$ denote the set of $p \times p$ matrices $g=(g(i, j))_{1 \leq i, j \leq p}$ with positive coefficients such that for any $1 \leq i, j, k, l \leq p$,

$$
\frac{1}{B} \leq \frac{g(i, j)}{g(k, l)} \leq B
$$

On the product space $\mathbb{X} \times S^{+}$, we define the function $\rho$ by setting $\rho(\tilde{x}, g):=$ $\log |\tilde{x} g|$ for $(\tilde{x}, g) \in \mathbb{X} \times S^{+}$. This function satisfies the co-cycle property, namely for any $g, h \in S^{+}$and $\tilde{x} \in \mathbb{X}$,

$$
\rho(\tilde{x}, g h)=\rho(\tilde{x} \cdot g, h)+\rho(\tilde{x}, g) .
$$

Under hypothesis H3 introduced below, there exists a unique $\mu$-invariant measure $v$ on $\mathbb{X}$ such that for any continuous function $\varphi$ on $\mathbb{X}$,

$$
(\mu * v)(\varphi)=\int_{S^{+}} \int_{\mathbb{X}} \varphi(\tilde{x} \cdot g) v(d \tilde{x}) \mu(d g)=\int_{\mathbb{X}} \varphi(\tilde{x}) v(d \tilde{x})=v(\varphi) .
$$

Moreover, the upper Lyapunov exponent $\pi_{\mu}$ defined above coincides with the quantity $\int_{\mathbb{X} \times S^{+}} \rho(\tilde{x}, g) \mu(d g) v(d \tilde{x})$ and is finite [5].

In the sequel, we first focus our attention on "linear-fractional multidimensional generating functions" $f=f_{\gamma, M}$ of the form

$$
f(s)=f_{\gamma, M}(s)=\mathbf{1}-\frac{1}{1+\tilde{\gamma}(\mathbf{1}-s)} M(\mathbf{1}-s),
$$


for any $s \in[0,1]^{p}$, where $\tilde{\gamma}=(\gamma, \ldots, \gamma) \in \mathbb{R}^{p}$ with $\gamma>0$ and $M \in S^{+}$. For such a function $f=f_{\gamma, M}$, we set $\gamma=\gamma(f)$ and $M=M(f)$ and notice that $M(f)$ equals the mean matrix $\left(\frac{\partial f^{(i)}}{\partial s_{j}}(\mathbf{1})\right)_{1 \leq i, j \leq p}$.

Here, we specify hypotheses concerning the distribution $\mu$ of the mean matrices $M_{\xi_{n}}=M\left(f_{\xi_{n}}\right), n \geq 1$.

H1. There exists $\epsilon_{0}>0$ such that $\int_{S^{+}} N(g)^{\epsilon_{0}} \mu(d g)<\infty$.

$\mathrm{H} 2$. (Strong irreducibility). There exists no affine subspaces $\mathcal{A}$ of $\mathbb{R}^{d}$ such that $\mathcal{A} \cap \mathcal{C}$ is nonempty, bounded and invariant under the action of all elements of the support of $\mu$.

H3. There exists $B \geq 1$ such that $\mu\left(S^{+}(B)\right)=1$.

H4. The upper Lyapunov exponent $\pi_{\mu}$ of $\mu$ is equal to 0 .

H5. There exists $\delta>0$ such that $\mu\left(E_{\delta}\right)>0$, where

$$
E_{\delta}:=\left\{g \in S^{+}|\forall \tilde{x} \in \mathbb{X}, \log | \tilde{x} g \mid \geq \delta\right\} .
$$

If the variables $f_{\xi_{n}}$ are linear fractional generating functions, then we introduce an additional hypothesis.

H6. There exists $B^{\prime} \geq 1$ such that $\frac{1}{B^{\prime}} \leq \gamma\left(f_{\xi_{n}}\right) \leq B^{\prime} \mathbb{P}$-a.s.

Here comes the main result of this paper.

THEOREM 1.1. Assume that the random variables $f_{\xi_{n}}$ are linear fractional generating functions and that hypotheses $\mathrm{H} 1-\mathrm{H} 6$ hold. Then, for any $i \in\{1, \ldots, p\}$, there exists a real number $\beta_{i} \in(0,+\infty)$ such that

$$
\lim _{n \rightarrow+\infty} \sqrt{n} \mathbb{P}\left(Z_{n} \neq \tilde{\mathbf{0}} \mid Z_{0}=\tilde{e}_{i}\right)=\beta_{i}
$$

For general generating functions, we obtain the following weaker result.

THEOREM 1.2. Assume that the random variables $f_{\xi_{n}}$ are $C^{2}$-functions on $[0,1]^{p}$ such that:

1. There exists $A>0$ such that for any $i, k, l \in\{1, \ldots, p\}$,

$$
\frac{\partial^{2} f_{\xi_{n}}^{(i)}}{\partial s_{k} \partial s_{l}}(\mathbf{1}) \leq A \frac{\partial f_{\xi_{n}}^{(i)}}{\partial s_{k}}(\mathbf{1}) \quad \mathbb{P} \text {-a.s. }
$$

2. The distribution $\mu$ of the matrices $M_{\xi_{n}}=\left(\frac{\partial f_{\xi_{n}}^{(i)}}{\partial s_{j}}(\mathbf{1})\right)_{1 \leq i, j \leq p}$ satisfies hypotheses $\mathrm{H} 1-\mathrm{H} 5$.

Then there exist real constants $0<c_{1}<c_{2}<+\infty$ such that for any $i \in\{1, \ldots, p\}$, and $n \geq 1$,

$$
\frac{c_{1}}{\sqrt{n}} \leq \mathbb{P}\left(Z_{n} \neq \tilde{\mathbf{0}} \mid Z_{0}=\tilde{e}_{i}\right) \leq \frac{c_{2}}{\sqrt{n}}
$$


In particular, under weaker assumptions than in [18], this theorem states that the process $\left(Z_{n}\right)_{n \geq 0}$ becomes extinct $\mathbb{P}$-a.s. in the critical case. Notice that by a recent work [8], equivalence (1.6) holds true even when the $f_{\xi_{n}}$ are not assumed to be linear fractional generating functions. The authors in [8] apply the proof in the present paper with two new ingredients: first, an extension to the multi-type case of Geiger and Kersting's decomposition of the extinction probability ([10], formula (2.2)) and second, a variation of Lemma 3.1 below, taking into account the residual term which appears in this expression of the extinction probability.

Notation. Let $c>0$ and $\phi, \psi$ be two functions of some variable $x$; we shall write $\phi \stackrel{c}{\preceq} \psi$ (or simply $\phi \preceq \psi$ ) when $\phi(x) \leq c \psi(x)$ for any value of $x$. The notation $\phi \asymp$ c (or simply $\phi \asymp \psi$ ) means $\phi \stackrel{c}{\preceq} \psi \stackrel{c}{\preceq} \phi$.

\section{Preliminary results.}

2.1. Product of matrices with nonnegative coefficients. We describe in this section some properties of the set $S^{+}$. We first endow $\mathbb{X}$ with a distance $d$ which is a variant of the Hilbert metric; it is bounded on $\mathbb{X}$ and any element $g \in S^{+}$ acts on $(\mathbb{X}, d)$ as a contraction. We summarize here its construction and its major properties.

For any $\tilde{x}=\left(x_{i}\right)_{1 \leq i \leq p}, \tilde{y}=\left(y_{i}\right)_{1 \leq i \leq p} \in \mathbb{X}$, we write

$$
m(\tilde{x}, \tilde{y})=\min \left\{\frac{x_{i}}{y_{i}} \mid i=1, \ldots, p \text { such that } y_{i}>0\right\}
$$

and we set

$$
d(\tilde{x}, \tilde{y}):=\varphi(m(\tilde{x}, \tilde{y}) m(\tilde{y}, \tilde{x})),
$$

where $\varphi$ is the one-to-one function on $[0,1]$ defined by $\varphi(s):=\frac{1-s}{1+s}$. For $g \in S^{+}$, we set

$$
c(g):=\sup \{d(\tilde{x} \cdot g, \tilde{y} \cdot g) \mid \tilde{x}, \tilde{y} \in \mathbb{X}\} .
$$

We present some crucial properties of $d$.

PROpOsItion 2.1 ([17]). The function $d$ is a distance on $\mathbb{X}$ which satisfies the following properties:

1. $\sup \{d(\tilde{x}, \tilde{y}) \mid \tilde{x}, \tilde{y} \in \mathbb{X}\}=1$.

2. For any $g=(g(i, j))_{i, j} \in S^{+}$

$$
c(g)=\max _{i, j, k, l \in\{1, \ldots, p\}} \frac{|g(i, j) g(k, l)-g(i, l) g(k, j)|}{g(i, j) g(k, l)+g(i, l) g(k, j)} .
$$

In particular, there exists $\kappa_{B} \in[0,1)$ such that $c(g) \leq \kappa_{B}<1$ for any $g \in S^{+}(B)$. 
3. $d(\tilde{x} \cdot g, \tilde{y} \cdot g) \leq c(g) d(\tilde{x}, \tilde{y}) \leq c(g)$ for any $\tilde{x}, \tilde{y} \in \mathbb{X}$ and $g \in S^{+}(B)$.

4. $c\left(g g^{\prime}\right) \leq c(g) c\left(g^{\prime}\right)$ for any $g, g^{\prime} \in S^{+}(B)$.

The following lemma follows from [9], Lemma 2. It is the key argument in the sequel to control the asymptotic behaviour of the norm of products of matrices of $S^{+}(B)$. Let $T_{S^{+}(B)}$ be the closed semi-group generated by the set $S^{+}(B)$.

LEMMA 2.1. For any $g \in T_{S^{+}(B)}$ and $1 \leq i, j, k, l \leq p$,

$$
g(i, j) \stackrel{B^{2}}{\asymp} g(k, l) .
$$

In particular, there exist $c>1$ such that for any $g, h \in T_{S^{+}(B)}$ and $\tilde{x}, \tilde{y} \in \mathbb{X}$ :

1. $|g x| \stackrel{c}{\asymp}|g|$ and $|\tilde{y} g| \stackrel{c}{\asymp}|g|$,

2. $|\tilde{y} g x| \stackrel{c}{\complement}|g|$,

3. $|g h| \asymp|g||h|$.

2.2. Conditioned product of random matrices. Recall that $\left(M_{n}\right)_{n \geq 0}$ is a sequence of i.i.d. matrices whose law $\mu$ satisfies hypotheses $\mathrm{H} 1-\mathrm{H} 5$ and $R_{n}=$ $M_{0} \ldots M_{n-1}$ and $L_{n}=M_{n-1} \ldots M_{0}$ for $n \geq 1$. Consider the homogenous Markov chain $\left(X_{n}\right)_{n \geq 0}$ on $\mathbb{X}$, with initial value $X_{0}=\tilde{x} \in \mathbb{X}$, defined by

$$
X_{n}=\tilde{x} \cdot R_{n}, \quad n \geq 1 .
$$

Its transition probability $P$ is given by: for any $\tilde{x} \in \mathbb{X}$ and any bounded Borel function $\varphi: \mathbb{X} \rightarrow \mathbb{R}$

$$
P \varphi(\tilde{x}):=\int_{S^{+}} \varphi(\tilde{x} \cdot g) \mu(d g) .
$$

The chain $\left(X_{n}\right)_{n \geq 0}$ has been the object of many studies, in particular there exists on $\mathbb{X}$ a unique $P$-invariant probability measure $v$.

Indeed, by Proposition 2.1, for any $\tilde{x}, \tilde{y} \in \mathbb{X}$ and any sequence $\left(g_{n}\right)_{n \geq 1}$ of elements in $S^{+}(B)$, it follows that

$$
d\left(\tilde{x} \cdot g_{n} \ldots g_{1}, \tilde{y} \cdot g_{n} \ldots g_{1}\right) \leq \kappa_{B}^{n} .
$$

This yields $\sup _{k \geq 0} d\left(\tilde{x} \cdot g_{n+k} \ldots g_{n} \ldots g_{1}, \tilde{x} \cdot g_{n} \ldots g_{1}\right) \rightarrow 0$ as $n \rightarrow+\infty$; the sequence $\left(\tilde{x} \cdot g_{1} \ldots g_{n}\right)_{n \geq 0}$ thus converges in $\mathbb{X}$.

In particular, under hypothesis $\mathrm{H} 3$, the sequence $\left(\tilde{x} \cdot L_{n}\right)_{n \geq 0}$ converges $\mathbb{P}$-a.s. towards some $\mathbb{X}$-valued random variable $X_{\infty}$. It follows that the Markov chain $\left(\tilde{x} \cdot R_{n}\right)_{n \geq 0}$ converges in distribution to the law $v$ of $X_{\infty}$, which is the unique $P$-invariant probability measure on $\mathbb{X}$. Property 2.2 allows one to prove that the restriction of $P$ to some suitable space of continuous functions from $\mathbb{X}$ to $\mathbb{C}$ is quasi-compact, which is a crucial ingredient to study the asymptotic behaviour of $\left(\tilde{x} \cdot R_{n}\right)_{n \geq 0}[5,14,17]$. 
In the sequel, we are interested in the left linear action $\tilde{x} \mapsto \tilde{x} R_{n}$ of the right products $R_{n}$, for any $\tilde{x} \in \mathbb{X}$. Since it holds

$$
\tilde{x} R_{n}=e^{\log \left|\tilde{x} R_{n}\right|} \tilde{x} \cdot R_{n},
$$

we consider the random process $\left(S_{n}\right)_{n \geq 0}$ defined by: for any $\tilde{x} \in \mathbb{X}, a \in \mathbb{R}$ and $n \geq 1$,

$$
S_{0}=S_{0}(\tilde{x}, a):=a \quad \text { and } \quad S_{n}=S_{n}(\tilde{x}, a):=a+\log \left|\tilde{x} R_{n}\right| .
$$

Iterating the co-cycle property (1.4), the basic decomposition of $S_{n}(\tilde{x}, a)$ arrives:

$$
S_{n}(\tilde{x}, a)=a+\log \left|\tilde{x} R_{n}\right|=a+\sum_{k=0}^{n-1} \rho\left(X_{k}, M_{k}\right) .
$$

Let us emphasize that for any $a \in \mathbb{R}$ the sequence $\left(X_{n}, S_{n}\right)_{n \geq 0}$ is a Markov chain on $\mathbb{X} \times \mathbb{R}$ whose transition probability $\widetilde{P}$ is defined by: for any $(\tilde{x}, a) \in \mathbb{X} \times \mathbb{R}$ and any bounded Borel function $\psi: \mathbb{X} \times \mathbb{R} \rightarrow \mathbb{C}$,

$$
\widetilde{P} \psi(\tilde{x}, a)=\int_{S^{+}} \psi(\tilde{x} \cdot g, a+\rho(\tilde{x}, g)) \mu(d g) .
$$

We set $\mathbb{R}_{*}^{+}:=\mathbb{R}^{+} \backslash\{0\}$ and denote $\widetilde{P}_{+}$the restriction of $\widetilde{P}$ to $\mathbb{X} \times \mathbb{R}^{+}$defined by: for $a \geq 0$ and any $\tilde{x} \in \mathbb{X}$,

$$
\widetilde{P}_{+}((\tilde{x}, a), \cdot)=1_{\mathbb{X} \times \mathbb{R}^{+}}(\cdot) \widetilde{P}((\tilde{x}, a), \cdot) .
$$

Fix $a \geq 0$ and denote by $\tau$ the first time greater than 1 the random process $\left(S_{n}\right)_{n \geq 0}$ becomes nonpositive:

$$
\tau:=\min \left\{n \geq 1 \mid S_{n} \leq 0\right\} .
$$

For any $\tilde{x} \in \mathbb{X}$ and $a \geq 0$, let us denote $\mathbb{P}_{\tilde{x}, a}$ the probability measure on $(\Omega, \mathcal{F}, \mathbb{P})$ conditioned to the event $\left[X_{0}=\tilde{x}, S_{0}=a\right]$ and $\mathbb{E}_{\tilde{x}, a}$ the corresponding expectation; we omit the index $a$ when $a=0$ and denote $\mathbb{P}_{\tilde{x}}$ the corresponding probability. For any $n \geq 0$, let $m_{n}=m_{n}(\tilde{x}, a)$ be the minimum of the values $S_{1}(\tilde{x}, a), \ldots, S_{n}(\tilde{x}, a)$ and set

$$
\mathbf{m}_{n}(\tilde{x}, a):=\mathbb{P}_{\tilde{x}, a}\left[m_{n}>0\right]=\mathbb{P}_{\tilde{x}, a}(\tau>n) .
$$

We present a general result concerning the behaviour of the tail distribution of the random variable $\tau$.

The asymptotic behaviour of the probability $\mathbb{P}_{\tilde{x}, a}(\tau>n)$ is established in [13] when the matrices $M_{n}$ are invertible and in [21] when the $M_{n}$ has nonnegative entries, under several conditions P1-P5; the first step is to establish the existence of a $\widetilde{P}_{+}$-harmonic function $h$ on $\mathbb{X} \times \mathbb{R}^{+}$. Our hypotheses $\mathrm{H} 1, \mathrm{H} 2, \mathrm{H} 4$ and H5 are exactly P1, P2, P4 and P5 in [21] and H3 is obviously stronger than P3. Proposition 2.2 concerns the existence of the function $h$ and its properties. 
Proposition 2.2 ([21]). Under hypotheses $\mathrm{H} 1-\mathrm{H} 5$, for any $x \in \mathbb{X}$ and $a \geq 0$, the sequence $\left(\mathbb{E}_{x, a}\left[S_{n} ; \tau>n\right]\right)_{n \geq 0}$ converges to a function $h(\tilde{x}, a)$ which satisfies the following properties:

1. For any $x \in \mathbb{X}$, the function $h(\tilde{x}, a)$ is increasing on $\mathbb{R}^{+}$.

2. There exists $c>0$ and $A>0$ such that for any $x \in \mathbb{X}$ and $a \geq 0$,

$$
\frac{1}{c} \vee(a-A) \leq h(\tilde{x}, a) \leq c(1+a) .
$$

2. For any $x \in \mathbb{X}$, the function $h(\tilde{x}, a)$ satisfies $\lim _{a \rightarrow+\infty} \frac{h(\tilde{x}, a)}{a}=1$.

3. The function $h$ is $\widetilde{P}_{+}$-harmonic.

This statement yields to the following theorem about the limit behaviour of $\mathbb{P}_{\tilde{x}, a}(\tau>n)$ as $n \rightarrow+\infty$; the relation $u_{n} \sim v_{n}$ defines $\lim _{n \rightarrow+\infty} \frac{u_{n}}{v_{n}}=1$.

THEOREM 2.1 ([21]). Under hypotheses $\mathrm{H} 1-\mathrm{H} 5$, for any $\tilde{x} \in \mathbb{X}$ and $a \geq 0$,

$$
\mathbb{P}_{\tilde{x}, a}(\tau>n) \sim \frac{2}{\sigma \sqrt{2 \pi n}} h(\tilde{x}, a) \quad \text { as } n \rightarrow \infty,
$$

where $\sigma^{2}>0$ is the variance of the Markov walk $\left(S_{n}\right)_{n \geq 0}$, given in [13]. Moreover, there exists $a$ constant $c>0$ such that for any $\tilde{x} \in \mathbb{X}, a \geq 0$ and $n \geq 0$,

$$
\sqrt{n} \mathbb{P}_{\tilde{x}, a}(\tau>n) \leq \operatorname{ch}(\tilde{x}, a) .
$$

REMARK. The fact that $\sigma^{2}>0$ is a direct consequence of hypotheses $\mathrm{H} 2$ and $\mathrm{H} 5$ (which implies in particular that the semi-group generated by the support of $\mu$ is unbounded); see [5], Chapter 6, Lemmas 5.2 and 5.3 and Section 8 for more details.

\section{Proof of Theorem 1.1.}

3.1. Expression of nonextinction probability. For any $0 \leq k<n$, set $R_{k, n}:=$ $M_{k} \ldots M_{n-1}$ and $R_{k, n}:=I$ otherwise. Let $Y_{k, n}:=R_{k, n} \cdot 1$. It is known (see Lemma 1 in [6]) that

$$
\left(q_{n}^{(i)}\right)^{-1}=\frac{1+\tilde{\gamma}_{0} M_{1} \ldots M_{n-1} \mathbf{1}+\tilde{\gamma}_{1} M_{2} \ldots M_{n-1} \mathbf{1}+\cdots+\tilde{\gamma}_{n-1} \mathbf{1}}{\tilde{e}_{i} R_{n} \mathbf{1}} .
$$

Since $\tilde{e}_{i} R_{k} R_{k, n} \mathbf{1}=\tilde{e}_{i} M_{0} \ldots M_{n-1} \mathbf{1}$ for any $1 \leq k \leq n$, we may rewrite (3.1) as

$$
\left(q_{n}^{(i)}\right)^{-1}=\frac{1}{\tilde{e}_{i} R_{n} \mathbf{1}}+\sum_{k=0}^{n-1} \frac{\tilde{\gamma}_{k} Y_{k+1, n}}{\tilde{e}_{i} R_{k} Y_{k+1, n}}=\frac{1}{\tilde{e}_{i} R_{n} \mathbf{1}}+\sum_{k=0}^{n-1} \frac{\gamma_{k}}{\tilde{e}_{i} R_{k} Y_{k+1, n}} .
$$


Notice that, for any fixed $k \geq 0$, the sequence $\left(Y_{k, n}\right)_{n \geq 0}$ converges to some limit $Y_{k, \infty}[9,17]$. Indeed, the matrices $M_{l}, l \geq 1$, all belong to $S^{+}(B)$, so do their transposes and we may write as in (2.2):

$$
\sup _{m \geq 1} d\left(\widetilde{Y}_{k, n}, \tilde{Y}_{k, n+m}\right) \leq \kappa_{B}^{n-k} \stackrel{n \rightarrow+\infty}{\longrightarrow} 0 .
$$

The sequence of row vectors $\left(\tilde{Y}_{k, n}\right)_{n \geq 0}$ is thus a Cauchy sequence in $\mathbb{X}$, it converges and so does $\left(Y_{k, n}\right)_{n \geq 0}$; we denote $Y_{k, \infty}$ the limit of this last sequence. Using this key fact, we prove in Lemma 3.2 that the sequence $\left(q_{n}^{(i)}\right)_{n \geq 1}$ converges to some positive limit

$$
q_{\infty}^{(i)}=\left(\sum_{k=0}^{+\infty} \frac{\gamma_{k}}{\tilde{e}_{i} R_{k} Y_{k+1, \infty}}\right)^{-1}
$$

in the $L^{1}$-norm with respect to a probability measure $\widehat{\mathbb{P}}_{\tilde{x}, a}$ introduced in the following subsection.

3.2. Construction of a new probability measure $\widehat{\mathbb{P}}_{\tilde{x}, a}$ conditioned to the environment. Since the function $h$ is $\widetilde{P}_{+}$-harmonic on $\mathbb{X} \times \mathbb{R}^{+}$, it gives rise to a Markov kernel $\widetilde{P}_{+}^{h}$ on $\mathbb{X} \times \mathbb{R}^{+}$defined by

$$
\widetilde{P}_{+}^{h} \phi=\frac{1}{h} \widetilde{P}_{+}(h \phi)
$$

for any bounded measurable function $\phi$ on $\mathbb{X} \times \mathbb{R}^{+}$. The kernels $\widetilde{P}_{+}$and $\widetilde{P}_{+}^{h}$ are related to the stopping times $\tau$ by the following identity: for any $\tilde{x} \in \mathbb{X}, a \geq 0$ and $n \geq 1$,

$$
\begin{aligned}
\left(\widetilde{P}_{+}^{h}\right)^{n} \phi(\tilde{x}, a) & =\frac{1}{h(\tilde{x}, a)} \widetilde{P}_{+}^{n}(h \phi)(\tilde{x}, a) \\
& =\frac{1}{h(\tilde{x}, a)} \mathbb{E}_{\tilde{x}, a}\left(h \phi\left(X_{n}, S_{n}\right) ; \tau>n\right) \\
& =\frac{1}{h(\tilde{x}, a)} \mathbb{E}_{\tilde{x}, a}\left(h \phi\left(X_{n}, S_{n}\right) ; m_{n}>0\right) .
\end{aligned}
$$

This new Markov chain with kernel $\widetilde{P}_{+}^{h}$ allows one to change the measure on the canonical path space $\left((\mathbb{X} \times \mathbb{R})^{\otimes \mathbb{N}}, \sigma\left(X_{n}, S_{n}: n \geq 0\right), \theta\right)$ of the Markov chain $\left(X_{n}, S_{n}\right)_{n \geq 0}{ }^{1}$ from $\mathbb{P}$ to the measure $\widehat{\mathbb{P}}_{\tilde{x}, a}$ characterized by the property that

$$
\begin{aligned}
\widehat{\mathbb{E}}_{\tilde{x}, a} & {\left[\varphi\left(X_{0}, S_{0}, \ldots, X_{k}, S_{k}\right)\right] } \\
& =\frac{1}{h(\tilde{x}, a)} \mathbb{E}_{\tilde{x}, a}\left[\varphi\left(X_{0}, \ldots, S_{k}\right) h\left(X_{k}, S_{k}\right) ; m_{k}>0\right]
\end{aligned}
$$

for any positive Borel function $\varphi$ on $(\mathbb{X} \times \mathbb{R})^{k+1}$.

\footnotetext{
${ }^{1} \theta$ denotes the shift operator on $(\mathbb{X} \times \mathbb{R})^{\otimes \mathbb{N}}$ defined by $\theta\left(\left(x_{k}, s_{k}\right)_{k \geq 0}\right)=\left(x_{k+1}, s_{k+1}\right)_{k \geq 0}$ for any $\left(x_{k}, s_{k}\right)_{k \geq 0}$ in $(\mathbb{X} \times \mathbb{R})^{\otimes \mathbb{N}}$.
} 
For any $0 \leq k \leq n$,

$$
\begin{aligned}
\mathbb{E}_{\tilde{x}, a}[ & \left.\varphi\left(X_{0}, S_{0}, \ldots, X_{k}, S_{k}\right) ; m_{n}>0\right] \\
= & \mathbb{E}_{\tilde{x}, a}\left[\varphi\left(X_{0}, \ldots, S_{k}\right) ; S_{1}>0, \ldots, S_{n}>0\right] \\
= & \mathbb{E}_{\tilde{x}, a}\left[\varphi\left(X_{0}, \ldots, S_{k}\right) ; a+\rho\left(X_{0}, M_{0}\right)>0,\right. \\
& \left.\ldots, a+\sum_{i=0}^{k-1} \rho\left(X_{i}, M_{i}\right)+\sum_{i=k}^{n-1} \rho\left(X_{i}, M_{i}\right)>0\right] \\
= & \mathbb{E}_{\tilde{x}, a}\left[\mathbb { E } \left[\varphi\left(X_{0}, \ldots, S_{k}\right) ; S_{1}>0, \ldots, S_{k}>0,\right.\right. \\
& \left.\left.S_{k}+S_{1} \circ \theta^{k}>0, \ldots, S_{k}+S_{n-k} \circ \theta^{k}>0 \mid M_{0}, \ldots, M_{k-1}\right]\right] \\
= & \mathbb{E}_{\tilde{x}, a}\left[\varphi\left(X_{0}, \ldots, S_{k}\right)\right. \\
& \left.\times \mathbb{E}\left[S_{k}+S_{1} \circ \theta^{k}>0, \ldots, S_{k}+S_{n-k} \circ \theta^{k}>0 \mid M_{0}, \ldots, M_{k-1}\right] ; m_{k}>0\right] \\
= & \mathbb{E}_{\tilde{x}, a}\left[\varphi\left(X_{0}, \ldots, S_{k}\right)\right. \\
& \left.\times \mathbb{P}_{X_{k}, S_{k}}\left(S_{1} \circ \theta^{k}>0, \ldots, S_{n-k} \circ \theta^{k}>0\right) ; m_{k}>0\right] \\
= & \mathbb{E}_{\tilde{x}, a}\left[\varphi\left(X_{0}, \ldots, S_{k}\right) \mathbb{P}_{X_{k}, S_{k}}(\tau>n-k) ; m_{k}>0\right] .
\end{aligned}
$$

Hence,

$$
\begin{aligned}
& \mathbb{E}_{\tilde{x}, a}\left(\varphi\left(X_{0}, \ldots, S_{k}\right) ; m_{n}>0\right) \\
& \quad=\mathbb{E}_{\tilde{x}, a}\left[\varphi\left(X_{0}, \ldots, S_{k}\right) \mathbf{m}_{n-k}\left(X_{k}, S_{k}\right) ; m_{k}>0\right] .
\end{aligned}
$$

Moreover, in view of Theorem 2.1, the dominated convergence theorem and (3.5), we obtain for any bounded function $\varphi$ with compact support,

$$
\begin{aligned}
& \lim _{n \rightarrow+\infty} \mathbb{E}_{\tilde{x}, a}\left[\varphi\left(X_{0}, \ldots, S_{k}\right) \mid m_{n}>0\right] \\
& \quad= \frac{1}{h(\tilde{x}, a)} \mathbb{E}_{\tilde{x}, a}\left[\varphi\left(X_{0}, \ldots, S_{k}\right) h\left(X_{k}, S_{k}\right) ; m_{k}>0\right] \\
& \quad=\widehat{\mathbb{E}}_{\tilde{x}, a}\left[\varphi\left(X_{0}, \ldots, S_{k}\right)\right]
\end{aligned}
$$

which clarifies the interpretation of $\widehat{\mathbb{P}}_{\tilde{x}, a}$.

We formalize in three steps the construction of a new probability measure, denoted again $\widehat{\mathbb{P}}_{\tilde{x}, a}$, for each $\tilde{x} \in \mathbb{X}$ and $a \geq 0$, but defined this time on the bigger $\sigma$-algebra $\sigma\left(f_{n}, Z_{n}: n \geq 0\right)$. Retaining the notation from the previous parts, the measure $\widehat{\mathbb{P}}_{\tilde{x}, a}$ is characterized by properties (3.4), (3.7) and (3.8).

Step 1 . The marginal distribution of $\widehat{\mathbb{P}}_{\tilde{x}, a}$ on $\sigma\left(X_{n}, S_{n}: n \geq 0\right)$ is $\widehat{\mathbb{P}}_{\tilde{x}, a}$ characterized by the property (3.4). 
Step 2. For any $n \geq 0$, the conditional distribution of $\left(f_{0}, \ldots, f_{n}\right)$ under $\widehat{\mathbb{P}}_{\tilde{x}, a}$ given $X_{0}=\tilde{x}_{0}=\tilde{x}, \ldots, X_{n}=\tilde{x}_{n}, S_{0}=s_{0}=a, \ldots, S_{n}=s_{n}$ is given by

$$
\begin{aligned}
& \widehat{\mathbb{P}}_{\tilde{x}, a}\left(f_{k} \in A_{k}, 0 \leq k \leq n \mid X_{i}=\tilde{x}_{i}, S_{i}=s_{i}, 0 \leq i \leq n\right) \\
& \quad=\mathbb{P}\left(f_{k} \in A_{k}, 0 \leq k \leq n \mid X_{i}=\tilde{x}_{i}, S_{i}(\tilde{x})=s_{i}, 0 \leq i \leq n\right),
\end{aligned}
$$

for any measurable sets $A_{0}, \ldots, A_{n}$ in $\mathcal{H}_{B}$ and almost all $\left(\tilde{x}_{i}\right)_{0 \leq i \leq n}$ and $\left(s_{i}\right)_{0 \leq i \leq n}$ with respect to the law of $\left(X_{0}, \ldots, X_{n}, S_{0}, \ldots, S_{n}\right)$ under $\mathbb{P}$ [and also under $\widehat{\mathbb{P}}_{\tilde{x}, a}$ since, by formula (3.4), the probability measure $\widehat{\mathbb{P}}_{\tilde{x}, a}$ is absolutely continuous with respect to $\mathbb{P}$ on each $\sigma$-algebra $\left.\sigma\left(X_{0}, \ldots, S_{n}\right)\right]$.

Step 3 . The conditional distribution of $\left(Z_{n}\right)_{n \geq 0}$ under $\widehat{\mathbb{P}}_{\tilde{x}, a}$ given $f_{0}^{(i)}, f_{1}, \ldots$ is the same as under $\mathbb{P}$, namely

$$
\begin{aligned}
& \widehat{\mathbb{E}}_{\tilde{x}, a}\left(s^{Z_{n}} \mid Z_{0}, \ldots, Z_{n-1}, f_{0}^{(i)}, f_{1}, \ldots, f_{n-1}\right) \\
& \quad=f_{n-1}(s)^{Z_{n-1}}=\mathbb{E}\left(s^{Z_{n}} \mid Z_{0}, \ldots, Z_{n-1}, f_{0}^{(i)}, f_{1}, \ldots, f_{n-1}\right) .
\end{aligned}
$$

3.3. Proof of Theorem 1.1. We follow J. Geiger and G. Kersting's approach. We fix $1 \leq i \leq p$. For any $\tilde{x} \in \mathbb{X}$ and $a \geq 0$, let us denote $\mathbb{P}_{\tilde{x}, a}^{(i)}$ the probability measure on $(\Omega, \mathcal{F}, \mathbb{P})$ conditioned to the event $\left[X_{0}=\tilde{x}, S_{0}=a, Z_{0}=\tilde{e}_{i}\right]$ and $\mathbb{E}_{\tilde{x}, a}^{(i)}$ the corresponding expectation.

First, notice that the quantity $\mathbb{P}\left(Z_{n} \neq \tilde{\mathbf{0}} \mid Z_{0}=\tilde{e}_{i}\right)$ equals $\mathbb{E}_{\tilde{x}, a}\left(q_{n}^{(i)}\right)$ for any $(x, a) \in \mathbb{X} \times \mathbb{R}^{+}$; thus we fix $(\tilde{x}, a) \in \mathbb{X} \times \mathbb{R}^{+}, \rho>1$ and $0 \leq k \leq n$ and decompose $\mathbb{P}\left(Z_{n} \neq \tilde{\mathbf{0}} \mid Z_{0}=\tilde{e}_{i}\right)$ as

$$
\mathbb{P}^{(i)}\left(Z_{n} \neq \tilde{\mathbf{0}}\right)=A_{n}+B_{n}+C_{n}-D_{n},
$$

where:

- $A_{n}=A_{n}(\tilde{x}, a)=\mathbb{P}_{\tilde{x}, a}^{(i)}\left(Z_{n} \neq \tilde{\mathbf{0}}, m_{n} \leq 0\right)$;

- $B_{n}=B_{n}(\tilde{x}, a, \rho)=\mathbb{P}_{\tilde{x}, a}^{(i)}\left(Z_{n} \neq \tilde{\mathbf{0}}, m_{n}>0\right)-\mathbb{P}_{\tilde{x}, a}^{(i)}\left(Z_{n} \neq \tilde{\mathbf{0}}, m_{\rho n}>0\right)$;

- $C_{n}=C_{n}(\tilde{x}, a, \rho, k)=\mathbb{P}_{\tilde{x}, a}^{(i)}\left(Z_{k} \neq \tilde{\mathbf{0}}, m_{\rho n}>0\right)$;

- $D_{n}=D_{n}(\tilde{x}, a, \rho, k)=\mathbb{P}_{\tilde{x}, a}^{(i)}\left(Z_{k} \neq \tilde{\mathbf{0}}, Z_{n}=\tilde{\mathbf{0}}, m_{\rho n}>0\right)$.

We decompose the proof in 5 steps and then conclude, letting first $k \rightarrow+\infty$, then $\rho \rightarrow 1$ and at last $a \rightarrow+\infty$ :

1. The quantity $A=A(\tilde{x}, a):=\limsup _{n \rightarrow+\infty} \sqrt{n} A_{n}(\tilde{x}, a)$ tends to 0 when $a \rightarrow+\infty$.

2. The quantity $B=B(\tilde{x}, a, \rho):=\limsup _{n \rightarrow+\infty} \sqrt{n} B_{n}(\tilde{x}, a, \rho)$ tends to 0 when $\rho \rightarrow+1$.

3. As $n \rightarrow+\infty$, the sequence $\left(\sqrt{n} C_{n}(\tilde{x}, a, \rho, k)\right)_{n \geq 0}$ converges to

$$
C=C(\tilde{x}, a, \rho, k):=c_{1} \frac{h(\tilde{x}, a)}{\sqrt{\rho}} \widehat{\mathbb{P}}_{x}\left(Z_{k} \neq \tilde{\mathbf{0}}\right) .
$$


4. For any $\rho>1$, the quantity $D=D(\tilde{x}, a, \rho, k):=\limsup _{n \rightarrow+\infty} \sqrt{n} \times$ $D_{n}(\tilde{x}, a, \rho, k)$ tends to 0 when $k \rightarrow+\infty$.

5. The sequence $\left(\widehat{\mathbb{P}}_{\tilde{x}, a}^{(i)}\left(Z_{k} \neq 0\right)\right)_{k \geq 0}$ converges to some limit $v^{(i)}(\tilde{x}, a)>0$.

Step 1 . We may write

$$
\begin{aligned}
A_{n}(\tilde{x}, a) & =\mathbb{E}_{\tilde{x}, a}\left(\mathbb{E}\left(Z_{n} \neq \tilde{\mathbf{0}} \mid f_{0}^{(i)}, f_{1}, \ldots, f_{n-1}\right) ; m_{n} \leq 0\right) \\
& =\mathbb{E}_{\tilde{x}, a}\left(q_{n}^{(i)} ; m_{n} \leq 0\right) .
\end{aligned}
$$

To control the quantity $q_{n}^{(i)}$, we use the expression (3.2); Lemma 2.1 yields

$$
\begin{aligned}
\frac{1}{q_{n}^{(i)}} & =\frac{1}{\tilde{e}_{i} R_{n} \mathbf{1}}+\sum_{k=0}^{n-1} \frac{\gamma_{k}}{\tilde{e}_{i} R_{k} Y_{k+1, n}} \\
& \geq \max _{0 \leq k \leq n-1}\left\{\frac{\gamma_{k}}{\widetilde{e}_{i} R_{k} Y_{k+1, n}}\right\} \varlimsup_{0 \leq k \leq n-1}\left\{\frac{1}{\left|\tilde{x} R_{k}\right|}\right\} \\
& \geq \frac{1}{\exp \left\{\min _{0 \leq k \leq n-1}\left(a+\ln \left|\tilde{x} R_{k}\right|\right)\right\}}
\end{aligned}
$$

so that $q_{n}^{(i)} \preceq \exp \left(m_{n}(\tilde{x}, a)\right)$ and by applying Theorem 2.1, equation (2.7), we obtain

$$
\begin{aligned}
A_{n}(\tilde{x}, a) & =\mathbb{P}_{\tilde{x}}^{(i)}\left(Z_{n} \neq \tilde{\mathbf{0}}, m_{n} \leq-a\right) \\
& \preceq \mathbb{E}_{\tilde{x}}\left[\exp \left(m_{n}\right) ; m_{n} \leq-a\right] \\
& \leq \sum_{k=a}^{+\infty} e^{-k} \mathbb{P}_{\tilde{x}}\left(-k<m_{n} \leq-k+1\right) \\
& \leq \sum_{k=a}^{+\infty} e^{-k} \mathbb{P}_{\tilde{x}, k}(\tau>n) \\
& \preceq \frac{1}{\sqrt{n}} \sum_{k=a}^{+\infty}(k+1) e^{-k}
\end{aligned}
$$

Notice that the sum $\sum_{k=a}^{+\infty}(k+1) e^{-k}$ becomes arbitrarily small for sufficiently great $a$. Consequently, $A(\tilde{x}, a)=\lim \sup _{n \rightarrow+\infty} \sqrt{n} A_{n}(\tilde{x}, a) \longrightarrow 0$ as $x \rightarrow+\infty$.

Step 2. Theorem 2.1 yields

$$
\begin{aligned}
0 \leq B_{n}(\tilde{x}, a, \rho) & =\mathbb{P}_{\tilde{x}, a}^{(i)}\left(Z_{n} \neq \tilde{\mathbf{0}}, m_{n}>0\right)-\mathbb{P}_{\tilde{x}, a}^{(i)}\left(Z_{n} \neq \tilde{\mathbf{0}}, m_{\rho n}>0\right) \\
& \sim c_{1} \frac{h(\tilde{x}, a)}{\sqrt{n}}\left(1-\frac{1}{\sqrt{\rho}}\right) \quad \text { as } n \rightarrow+\infty
\end{aligned}
$$


with $c_{1}=\frac{2}{\sigma \sqrt{2 \pi}}$. Hence

$$
B(\tilde{x}, a, \rho):=\limsup _{n \rightarrow+\infty} \sqrt{n} B_{n}(\tilde{x}, a, \rho) \leq c_{1} h(\tilde{x}, a)\left(1-\frac{1}{\sqrt{\rho}}\right) \stackrel{\rho \rightarrow 1}{\longrightarrow} 0 .
$$

Step 3. Fix $0 \leq k \leq n$. Using (3.5) and the fact that $1_{\left[m_{k}>0\right]}$ and $\mathbf{m}_{\rho n-k}\left(X_{k}, S_{k}\right)$ are measurable with respect to the $\sigma$-algebra $\sigma\left(f_{0}^{(i)}, f_{1}, \ldots, f_{k-1}\right)$, we may write

$$
\begin{aligned}
C_{n}(\tilde{x}, a, \rho, k) & =\mathbb{P}_{\tilde{x}, a}^{(i)}\left(Z_{k} \neq \tilde{\mathbf{0}}, m_{\rho n}>0\right) \\
& =\mathbb{P}_{\tilde{x}, a}^{(i)}\left(Z_{k} \neq \tilde{\mathbf{0}}, m_{k}>0, \mathbf{m}_{\rho n-k}\left(X_{k}, S_{k}\right)\right) \\
& =\mathbb{E}_{\tilde{x}, a}^{(i)}\left(\mathbb{E}\left(1_{Z_{k} \neq \tilde{\mathbf{0}}} 1_{\left[m_{k}>0\right]} \mathbf{m}_{\rho n-k}\left(X_{k}, S_{k}\right) \mid f_{0}^{(i)}, \ldots, f_{k-1}\right)\right) \\
& =\mathbb{E}_{\tilde{x}, a}^{(i)}\left(\mathbb{P}\left(Z_{k} \neq \tilde{\mathbf{0}} \mid f_{0}^{(i)}, \ldots, f_{k-1}\right) 1_{\left[m_{k}>0\right]} \mathbf{m}_{\rho n-k}\left(X_{k}, S_{k}\right)\right) \\
& =\mathbb{E}_{\tilde{x}, a}\left(q_{k}^{(i)}, m_{\rho n}>0\right) \\
& =\mathbb{E}_{\tilde{x}, a}\left(q_{k}^{(i)} \mid m_{\rho n}>0\right) \mathbb{P}_{\tilde{x}, a}\left(m_{\rho n}>0\right) .
\end{aligned}
$$

By (3.6), since $k$ is fixed

$$
\lim _{n \rightarrow+\infty} \mathbb{E}_{\tilde{x}, a}\left(q_{k}^{(i)} \mid m_{\rho n}>0\right)=\widehat{\mathbb{E}}_{\tilde{x}, a}\left(q_{k}^{(i)}\right)=\widehat{\mathbb{P}}_{\tilde{x}, a}^{(i)}\left(Z_{k} \neq \tilde{\mathbf{0}}\right) .
$$

Hence, by Theorem 2.1

$$
\lim _{n \rightarrow+\infty} \sqrt{n} C_{n}(\tilde{x}, a, \rho, k)=c_{1} \frac{h(\tilde{x}, a)}{\sqrt{\rho}} \widehat{\mathbb{P}}_{\tilde{x}, a}^{(i)}\left(Z_{k} \neq \tilde{\mathbf{0}}\right) .
$$

Step 4. As in the previous step, we may write

$$
\begin{aligned}
D_{n}(\tilde{x}, a, \rho, k) & =\mathbb{P}_{\tilde{x}, a}^{(i)}\left(Z_{k} \neq \tilde{\mathbf{0}}, Z_{n}=\tilde{\mathbf{0}}, m_{\rho n}>0\right) \\
& =\mathbb{P}_{\tilde{x}, a}^{(i)}\left(Z_{k} \neq \tilde{\mathbf{0}}, m_{\rho n}>0\right)-\mathbb{P}_{\tilde{x}, a}^{(i)}\left(Z_{n} \neq \tilde{\mathbf{0}}, m_{\rho n}>0\right) \\
& =\mathbb{E}_{\tilde{x}, a}\left(q_{k}^{(i)}, m_{\rho n}>0\right)-\mathbb{E}_{\tilde{x}, a}\left(q_{n}^{(i)}, m_{\rho n}>0\right) \\
& =\mathbb{E}_{\tilde{x}, a}\left(\left(q_{k}^{(i)}-q_{n}^{(i)}\right) \mathbf{m}_{(\rho-1) n}\left(X_{n}, S_{n}\right) ; m_{n}>0\right) \\
& \preceq \frac{1}{\sqrt{(\rho-1) n}} \frac{1}{h(\tilde{x}, a)} \mathbb{E}_{\tilde{x}, a}\left(\left(q_{k}^{(i)}-q_{n}^{(i)}\right) h\left(X_{n}, S_{n}\right) ; m_{n}>0\right) .
\end{aligned}
$$

Since $1_{\left[m_{n}>0\right]}$ and $h\left(X_{n}, S_{n}\right)$ are $\sigma\left(S_{0}, \ldots, S_{n}\right)$-measurable, we observe that

$$
\begin{aligned}
\sqrt{n} D_{n}(\tilde{x}, a, \rho, k) & \preceq \frac{1}{\sqrt{\rho-1}} \widehat{\mathbb{E}}_{\tilde{x}, a}\left(q_{k}^{(i)}-q_{n}^{(i)}\right) \\
& =\frac{1}{\sqrt{\rho-1}}\left(\widehat{\mathbb{P}}_{\tilde{x}, a}^{(i)}\left(Z_{k} \neq \tilde{\mathbf{0}}\right)-\widehat{\mathbb{P}}_{\tilde{x}, a}^{(i)}\left(Z_{n} \neq \tilde{\mathbf{0}}\right)\right) .
\end{aligned}
$$


Hence

$$
D(\tilde{x}, a, \rho, k)=\limsup _{n \rightarrow+\infty} \sqrt{n} D_{n}(\tilde{x}, a, \rho, k) \preceq \frac{1}{\sqrt{\rho-1}}\left(\widehat{\mathbb{P}}_{x}\left(Z_{k} \neq \tilde{\mathbf{0}}\right)-v^{(i)}(\tilde{x}, a)\right)
$$

so that $D(\tilde{x}, a, \rho, k) \longrightarrow 0$ as $k \rightarrow+\infty$.

Step 5. We first state the following lemmas whose proofs follow in the next section.

LEMMA 3.1. For any $\tilde{x} \in \mathbb{X}$ and $a \geq 0$,

$$
\widehat{\mathbb{E}}_{\tilde{x}, a} \sum_{n=0}^{+\infty} e^{-S_{n}}<+\infty
$$

This allows one to identify the limit of the sequence $\left(\widehat{\mathbb{P}}_{\tilde{x}, a}^{(i)}\left(Z_{k} \neq \tilde{\mathbf{0}}\right)\right)_{k \geq 0}$.

LEMMA 3.2. For any $\tilde{x} \in \mathbb{X}$ and $a \geq 0$, the sequence $\left(q_{n}^{(i)}\right)_{n \geq 1}$ converges to $q_{\infty}^{(i)}$ in $L^{1}\left(\widehat{\mathbb{P}}_{\tilde{x}, a}\right)$; in particular

$$
\lim _{k \rightarrow+\infty} \widehat{\mathbb{P}}_{\tilde{x}, a}^{(i)}\left(Z_{k} \neq \tilde{\mathbf{0}}\right)=\widehat{\mathbb{E}}_{\tilde{x}, a} q_{\infty}^{(i)},
$$

where $q_{\infty}^{(i)}$ is given by the expression (3.3).

From Lemma 3.2, it is obvious that $\widehat{\mathbb{E}}_{\tilde{x}, a} q_{\infty}^{(i)} \leq 1$. On the other hand, the expression of $q_{\infty}^{(i)}$ combined with Lemma 3.1 and the fact that $\left|\tilde{x} R_{n}\right| \asymp \tilde{e}_{i} R_{n} Y_{n+1, \infty}$ (see Lemma 2.1 property 2 ) yields $\widehat{\mathbb{E}}_{\tilde{x}, a} q_{\infty}^{(i)}>0$. In other words, for any $a \geq 0$,

$$
0<v^{(i)}(\tilde{x}, a):=\widehat{\mathbb{E}}_{\tilde{x}, a} q_{\infty}^{(i)}<+\infty .
$$

This achieves the proof of Step 5.

Let us complete the proof of Theorem 1.1. Letting $n \rightarrow+\infty$ in expression (3.9) yields, for any $\tilde{x} \in \mathbb{X}, a \geq 0, \rho>1$ and $k \geq 1$,

$$
C-D \leq \liminf _{n \rightarrow+\infty} \sqrt{n} \mathbb{P}^{(i)}\left(Z_{n} \neq \tilde{\mathbf{0}}\right) \leq \limsup _{n \rightarrow+\infty} \sqrt{n} \mathbb{P}^{(i)}\left(Z_{n} \neq \tilde{\mathbf{0}}\right) \leq A+B+C+D \text {. }
$$

Letting first $k \rightarrow+\infty$ then $\rho \rightarrow 1$ yields

$$
\begin{aligned}
c_{1} h(\tilde{x}, a) v^{(i)}(\tilde{x}, a) & \leq \liminf _{n \rightarrow+\infty} \sqrt{n} \mathbb{P}\left(Z_{n} \neq \tilde{\mathbf{0}}\right) \\
& \leq \limsup _{n \rightarrow+\infty} \sqrt{n} \mathbb{P}\left(Z_{n} \neq \tilde{\mathbf{0}}\right) \\
& \leq c_{1} h(\tilde{x}, a) v^{(i)}(\tilde{x}, a)+A(\tilde{x}, a) .
\end{aligned}
$$

Since $v^{(i)}(\tilde{x}, a)>0, h(\tilde{x}, a)>0$ and $A(\tilde{x}, a)<+\infty$, we obtain in particular

$$
0<\liminf _{n \rightarrow+\infty} \sqrt{n} \mathbb{P}\left(Z_{n} \neq \tilde{\mathbf{0}}\right) \leq \limsup _{n \rightarrow+\infty} \sqrt{n} \mathbb{P}\left(Z_{n} \neq \tilde{\mathbf{0}}\right)<+\infty .
$$


Finally, letting $a \longrightarrow+\infty$, we conclude that both limits

$$
\lim _{n \rightarrow+\infty} \sqrt{n} \mathbb{P}^{(i)}\left(Z_{n} \neq \tilde{\mathbf{0}}\right) \quad \text { and } \quad \lim _{a \rightarrow+\infty} c_{1} h(\tilde{x}, a) v^{(i)}(\tilde{x}, a)
$$

exist, coincide and belong to $] 0,+\infty[$.

3.4. Proof of Theorem 1.2. First, for any $n \geq 1$ and $s=\left(s_{1}, \ldots, s_{p}\right)$, we denote $F_{n}(s)=f_{\xi_{0}}\left(f_{\xi_{1}}\left(\ldots\left(f_{\xi_{n-1}}(s)\right) \ldots\right)\right)$. By definition of $q_{n}^{(i)}$, we have for any $0 \leq k<n$,

$$
q_{n}^{(i)}=\tilde{e}_{i}\left(F_{k}(\mathbf{1})-F_{k}(z)\right),
$$

where $z=z(k, n)=f_{\xi_{k}}\left(\ldots\left(f_{\xi_{n-1}}(0)\right) \ldots\right)$; the mean value theorem yields

$$
q_{n}^{(i)}=\tilde{e}_{i}\left(F_{k}(\mathbf{1})-F_{k}(z)\right) \leq \tilde{e}_{i} M_{0} \ldots M_{k-1} \mathbf{1} \asymp\left|\tilde{x} R_{k}\right|=\exp \left(S_{k}(\tilde{x}, 0)\right)
$$

so that $q_{n}^{(i)} \preceq \exp \left(m_{n}(\tilde{x}, 0)\right)$ and $\mathbb{E}\left[q_{n}^{(i)}\right] \preceq \mathbb{E}\left[e^{m_{n}(\tilde{x}, 0)}\right]=\mathbb{E}_{\tilde{x}}\left[e^{m_{n}}\right]$.

Using the same trick like in (3.10), we can deduce that there exists a constant $c_{2}$ such that

$$
\mathbb{E}_{\tilde{x}}\left[e^{m_{n}}\right]=\mathbb{E}_{\tilde{x}}\left[e^{m_{n}} ; m_{n} \leq 0\right] \sim \frac{c_{2}}{\sqrt{n}},
$$

and thus the upper estimate in equation (1.7) arrives.

To obtain the lower estimate in (1.7), for any $\mathbb{R}$-valued multidimensional generating function $f(s), s=\left(s_{1}, \ldots, s_{p}\right)^{T}$, we obtain (see, for instance, formulas (64) and (65) in [23])

$$
f(s) \leq 1-\left(\sum_{i=1}^{p} \frac{\partial f}{\partial s_{i}}(\mathbf{1})\left(1-s_{i}\right)\right)\left(1+\frac{\sum_{i, j=1}^{p} \frac{\partial^{2} f}{\partial s_{i} \partial s_{j}}(\mathbf{1})\left(1-s_{j}\right)\left(1-s_{i}\right)}{\sum_{l=1}^{p} \frac{\partial f}{\partial s_{l}}(\mathbf{1})\left(1-s_{l}\right)}\right)^{-1} .
$$

We set $g_{\xi_{n}}(s)=\mathbf{1}-\frac{M_{\xi_{\xi}}(\mathbf{1}-s)}{1+\tilde{\gamma}_{\xi_{n}}(\mathbf{1}-s)}$, where $M_{\xi_{n}}$ is the mean matrix of $f_{\xi_{n}}(s)$ and $\widetilde{\gamma}_{\xi_{n}}=$ $(A, \ldots, A)$; thus, hypothesis H6 holds for $g_{\xi_{n}}$ with $B^{\prime}=A$. Applying inequality (3.12) with $f=f_{\xi_{n}}^{(i)}$, we may write

$$
f_{\xi_{n}}^{(i)}(s) \leq g_{\xi_{n}}^{(i)}(s), \quad i=1, \ldots, p,
$$

which yields

$$
\mathbb{E}\left[\mathbf{1}-g_{\xi_{0}}\left(g_{\xi_{1}}\left(\ldots\left(g_{\xi_{n}-1}(0)\right) \ldots\right)\right)\right] \leq \mathbb{E}\left[\mathbf{1}-f_{0}\left(f_{1}\left(\ldots\left(f_{n-1}(0)\right) \ldots\right)\right)\right] .
$$

The lower estimate in equation (1.6) appears by applying Theorem 1.1 to the lefthand side of equation (3.13). Therefore, the assertion of Theorem 1.2 arrives.

4. Proof of lemmas. We first give some hints for the proof of Lemma 2.1 and describe later the proofs of Lemmas 3.1 and 3.2. 
4.1. Proof of Lemma 2.1. First, using (2.1), we obtain

$$
|g|=\sum_{i, j=1}^{p} g(i, j) \stackrel{p^{2} B^{2}}{\asymp} g(k, l) .
$$

Further properties can be easily deduced from (4.1). Indeed, the assertions we need are obvious by noticing that

$$
\begin{aligned}
& |g x|=\sum_{i, j=1}^{p} g(i, j) x_{j}{ }^{p^{3} B^{2}}|g|, \\
& \tilde{y} g x=\sum_{i, j=1}^{p} y_{i} g(i, j) x_{j}{\stackrel{p}{{ }^{2} B^{2}}}^{2}|g|, \\
& |g h|=\sum_{i, j, k=1}^{p} g(i, j) h(j, k){ }^{p^{7} B^{4}}|g||h| .
\end{aligned}
$$

4.2. Proof of Lemma 3.1. Before going into the proof, we first claim that in the critical case, for any $\delta>0$ and $c$ given from Lemma 2.1, there exists an integer $\kappa=\kappa(\delta, c) \geq 1$ such that

$$
\mu^{* \kappa}\left(E_{\delta}\right):=\mu^{* \kappa}\{g|\forall \tilde{x} \in \mathbb{X}, \log | \tilde{x} g \mid \geq \delta\}>0 .
$$

Indeed, let $\tau^{\prime}:=\inf \left\{n \geq 1|\log | R_{n} \mid \geq \log c+\delta\right\}$. The random variable $\tau^{\prime}$ is a stopping time with respect to the natural filtration $\left(\sigma\left(M_{0}, \ldots, M_{k}\right)\right)_{k \geq 0}$; furthermore, $\mathbb{P}\left(\tau^{\prime}<+\infty\right)=1$ since the Lyapunov exponent $\pi_{\mu}$ equals 0 , which yields

$$
\limsup _{n \rightarrow+\infty} \log \left|R_{n}\right|=\limsup _{n \rightarrow+\infty} \log \left|\tilde{x} \cdot R_{n}\right|=+\infty
$$

(this last property is a direct consequence of the ergodic theorem applied here to the Birkhof sums $\log \left|\tilde{x} \cdot R_{n}\right|, n \geq 0$ [5]).

Therefore, for any $\delta>0$ and $c$ given from Lemma 2.1, there exists $\kappa \geq 1$ such that $\mathbb{P}\left(\tau^{\prime}=\kappa\right)>0$. Moreover, we also have

$$
\begin{aligned}
\mathbb{P}\left(\log \left|R_{\kappa}\right| \geq \log c+\delta\right) & \geq \mathbb{P}\left(\log \left|R_{\kappa}\right| \geq \log c+\delta, \tau^{\prime}=\kappa\right) \\
& =\mathbb{P}\left(\log \left|R_{\tau^{\prime}}\right| \geq \log c+\delta, \tau^{\prime}=\kappa\right) \\
& =\mathbb{P}\left(\tau^{\prime}=\kappa\right)>0 .
\end{aligned}
$$

Since for any $\tilde{x} \in \mathbb{X}, g \in S^{+}(B),|g x| \geq \frac{|g|}{c}$, it follows that

$$
\{g|\log | g \mid \geq \log c+\delta\} \subset\{g|\forall \tilde{x} \in \mathbb{X}, \log | \tilde{x} g \mid \geq \delta\} .
$$

Thus,

$$
\begin{aligned}
0 & <\mathbb{P}\left(\log \left|R_{\kappa}\right| \geq \log c+\delta\right)=\mu^{* \kappa}\{g|\log | g \mid \geq \log c+\delta\} \\
& \leq \mu^{* \kappa}\{g|\forall \tilde{x} \in \mathbb{X}, \log | \tilde{x} g \mid \geq \delta\},
\end{aligned}
$$

which is the assertion of the claim (4.2). 
Let us go into the proof of Lemma 3.1. For any $\tilde{x} \in \mathbb{X}, a \geq 0$ and $\lambda \in(0,1)$, there exists some constant $C(\lambda)>0$ such that $(t+1) e^{-t} \leq C(\lambda) e^{-\lambda t}$ for any $t>0$. Hence

$$
\begin{aligned}
\widehat{\mathbb{E}}_{\tilde{x}, a}\left[\sum_{n=0}^{+\infty} e^{-S_{n}}\right] & \leq 1+\frac{1}{h(\tilde{x}, a)} \sum_{n=1}^{+\infty} \mathbb{E}_{\tilde{x}, a}\left(e^{-S_{n}} h\left(X_{n}, S_{n}\right) ; S_{1}>0, \ldots, S_{n}>0\right) \\
& \leq 1+\frac{c}{h(\tilde{x}, a)} \sum_{n=1}^{+\infty} \mathbb{E}_{\tilde{x}, a}\left(e^{-S_{n}}\left(1+S_{n}\right) ; S_{1}>0, \ldots, S_{n}>0\right) \\
& \leq 1+\frac{c C(\lambda)}{h(\tilde{x}, a)} \sum_{n=1}^{+\infty} \mathbb{E}_{\tilde{x}, a}\left(e^{-\lambda S_{n}} ; S_{1}>0, \ldots, S_{n}>0\right)
\end{aligned}
$$

where $c$ is introduced in inequality (2.5).

We define a function $\Phi$ for any $\tilde{x} \in \mathbb{X}$ and $a \in \mathbb{R}$ as follows:

$$
\Phi(\tilde{x}, a):=\sum_{n=1}^{+\infty} \mathbb{E}_{\tilde{x}, a}\left(e^{-\lambda S_{n}} ; S_{1}>0, \ldots, S_{n}>0\right) .
$$

Notice that for any $\tilde{x} \in \mathbb{X}$, the function $\Phi(\tilde{x}, \cdot)$ increases on $\mathbb{R}$. To prove Lemma 3.1, it is sufficient to check that for any $\tilde{x} \in \mathbb{X}$ and $a \in \mathbb{R}$

$$
\Phi(\tilde{x}, a)<+\infty .
$$

Let us explain briefly the strategy of our proof, inspired by Lemma 3.2 in [10]. J. Geiger and G. Kersting first use in a crucial way the so-called "duality principle" [i.e., the fact that for a classical random walk $\left(S_{n}\right)_{n \geq 1}$ on $\mathbb{R}$ the vectors $\left(S_{1}, S_{2}, \ldots, S_{n}\right)$ and $\left(S_{n}-S_{n-1}, S_{n}-S_{n-2}, \ldots, S_{n}\right)$ have the same distribution] and they prove in this context of random walks with i.i.d. increments that the quantity $\sum_{n=1}^{+\infty} \mathbb{E}_{a}\left[e^{-\lambda S_{n}} ; S_{1}>0, \ldots, S_{n}>0\right]$ is finite when $a=0$. Second, they extend this property for any $a \geq 0$.

In the multidimensional case, it is more complicated to apply the duality principle and we can only prove at the beginning that the quantity $\Phi\left(\tilde{x}, a_{0}\right)$ is finite for some $a_{0}<0$ (without any control on the value of $a_{0}$ ). To extend this property to $\Phi(\tilde{x}, a)$ for $a>a_{0}$, we use the crucially hypothesis H5; we refer to Step 2 below, especially inequality (4.5), and emphasize that the argument holds only when $-a_{0} \leq \delta$. To avoid this difficulty, we modify the function $\Phi$ by introducing the functions $\Phi_{\kappa}$ associated with the $\kappa$ th power of convolution $\mu^{* \kappa}$ of $\mu$. For any $\tilde{x} \in \mathbb{X}, a \in \mathbb{R}$, let

$$
\Phi_{\kappa}(\tilde{x}, a):=\sum_{n=1}^{+\infty} \mathbb{E}_{\tilde{x}, a}\left(e^{-\lambda S_{n \kappa}} ; S_{\kappa}>0, \ldots, S_{n \kappa}>0\right) .
$$

The relation is that $\Phi(\tilde{x}, a) \preceq \Phi_{\kappa}(\tilde{x}, a)$ for any $\tilde{x} \in \mathbb{X}, a \in \mathbb{R}$. Then, by using the duality principle, we bound from above $\Phi_{\kappa}(\tilde{x}, a)$ by a new quantity $\Psi_{\kappa}(\tilde{x})$ defined 
below for any $\tilde{x} \in \mathbb{X}$ and $a \in \mathbb{R}$. Finally, we prove $\Psi_{\kappa}(\tilde{x})<+\infty$ by using the ascending ladder epochs associated to the Markov walk $\left(L_{n} \cdot x, \log \left|L_{n} x\right|\right)_{n \geq 0}$ and the elementary renewal theorem.

We set $L_{0}=0$ and denote $L_{n}:=M_{n-1} \ldots M_{0}$ the left product of the matrices $M_{0}, \ldots, M_{n}$ when $n \geq 1$. Set, for any $\tilde{x} \in \mathbb{X}$,

$$
\Psi_{\kappa}(\tilde{x}):=\sum_{n=1}^{+\infty} \mathbb{E}\left[\left|L_{n \kappa} x\right|^{-\lambda} ;\left|L_{n \kappa} x\right|>\left|L_{(n-1) \kappa} x\right|, \ldots,\left|L_{n \kappa} x\right|>1\right] .
$$

Property (4.4) is a direct consequence of the four following steps:

1. For any $\kappa \geq 1$, there exists $C(\kappa)>0$ such that, for any $\tilde{x} \in \mathbb{X}$ and $a \in \mathbb{R}$,

$$
\Phi(\tilde{x}, a) \leq C(\kappa)\left(1+\Phi_{\kappa}(\tilde{x}, a)\right) .
$$

2. If there exist some $\kappa \geq 1, \tilde{x}_{0} \in \mathbb{X}$ and $a_{0}<0$ such that $0<\Phi_{\kappa}\left(\tilde{x}_{0}, a_{0}\right)<$ $+\infty$, then

$$
\forall \tilde{x} \in \mathbb{X}, \forall a \in \mathbb{R} \quad \Phi_{\kappa}(\tilde{x}, a)<+\infty .
$$

3. There exist $C_{1}>0$ and $a_{1}<0$ such that for any $\kappa \geq 1, \tilde{x} \in \mathbb{X}$ and $a<a_{1}$

$$
\Phi_{\kappa}(\tilde{x}, a) \stackrel{C_{1}}{\preceq} \Psi_{\kappa}(\tilde{x}) .
$$

4. For any $\kappa \geq 1$ and $\tilde{x} \in \mathbb{X}$,

$$
\Psi_{\kappa}(\tilde{x})<+\infty .
$$

With these steps at hand, we may prove (4.4) as follows. First, for any $a_{0} \leq$ $a_{1}<0$, we choose some $\delta_{0}$ such that $\delta_{0}>-a_{0}>0$. For each $\delta_{0}$, there exists $\kappa_{0} \geq 1$ such that $\mathbb{P}\left(\log \left|\tilde{x} R_{\kappa_{0}}\right| \geq \delta_{0}\right)>0$ [see (4.2) above]. Since $\delta_{0}>-a_{0}$, we have $\mathbb{P}_{\tilde{x}, a}\left(S_{\kappa_{0}}>0\right)>0$, which implies $\Phi_{\kappa_{o}}\left(\tilde{x}_{0}, a_{0}\right)>0$. On the other hand, since $a_{0} \leq a_{1}$, Step 3 and Step 4 yield $\Phi_{\kappa_{o}}\left(\tilde{x}_{0}, a_{0}\right)<+\infty$. Therefore, we can apply Step 2 and it yields $\Phi_{\kappa}(\tilde{x}, a)<+\infty$ for any $\tilde{x} \in \mathbb{X}$ and $a \in \mathbb{R}$. Finally, thanks to Step 1, (4.4) arrives.

Step 1 . It is easy to see that

$$
\begin{aligned}
\Phi(\tilde{x}, a) \leq & \sum_{r=1}^{\kappa-1} \mathbb{E}_{\tilde{x}, a}\left[e^{-\lambda S_{r}}\right]+\sum_{n=1}^{+\infty \kappa-1} \sum_{r=0}^{\kappa} \mathbb{E}_{\tilde{x}, a}\left[e^{-\lambda S_{n \kappa+r}} ; S_{\kappa}>0, \ldots, S_{n \kappa}>0\right] \\
\leq & \sum_{r=1}^{\kappa-1} \mathbb{E}_{\tilde{x}, a}\left(e^{-\lambda S_{r}}\right) \\
& +\sum_{n=1}^{+\infty} \mathbb{E}_{\tilde{x}, a}\left(e^{-\lambda S_{n \kappa}} ; S_{\kappa}>0, \ldots, S_{n \kappa}>0\right) \times \sum_{r=0}^{\kappa-1} \sup _{\tilde{y} \in \mathbb{X}} \mathbb{E}_{\tilde{y}, a}\left(e^{-\lambda S_{r}}\right) \\
\leq & \left(\sum_{r=0}^{\kappa-1} \sup _{\tilde{y} \in \mathbb{X}} \mathbb{E}_{\tilde{y}, a}\left(e^{-\lambda S_{r}}\right)\right)\left(1+\Phi_{\kappa}(\tilde{x}, a)\right),
\end{aligned}
$$


which yields to the expected result with

$$
0<C(\kappa)=\sum_{r=0}^{\kappa-1} \sup _{\tilde{y} \in \mathbb{X}} \mathbb{E}_{\tilde{y}, a}\left[e^{-\lambda S_{r}}\right]<+\infty .
$$

Step 2. The inequality $\Phi_{\kappa}\left(\tilde{x}_{0}, a_{0}\right)>0$ implies that $\mathbb{P}\left(\log \left|\tilde{x}_{0} R_{\kappa}\right|>-a_{0}\right)>0$; we thus fix $\delta>-a_{0}>0$ and $\kappa \geq 1$ such that $\mu^{* \kappa}\left(E_{\delta}\right)>0$. Since $a_{0}<0$, this property may hold only when $\kappa$ is large enough; this happens, for instance, when the support of $\mu$ is bounded.

To simplify the notation, we assume that $-a_{0}<\delta$ where $\delta$ is given by $\mathrm{H} 5$. We set $\kappa=1$ and write

$$
\begin{aligned}
\Phi\left(\tilde{x}_{0}, a_{0}\right)= & \sum_{n=1}^{+\infty} \mathbb{E}\left[\left|\tilde{x}_{0} R_{n}\right|^{-\lambda} ;\left|\tilde{x}_{0} R_{1}\right|>e^{-a_{0}}, \ldots,\left|\tilde{x}_{0} R_{n}\right|>e^{-a_{0}}\right] \\
\geq & \int_{\left\{g \in S^{+}(B):\left|\tilde{x}_{0} g\right| \geq e^{\left.-a_{0}\right\}}\right.} \sum_{n=2}^{+\infty} \mathbb{E}\left[\left|\tilde{x}_{0} g R_{1, n}\right|^{-\lambda} ;\left|\tilde{x}_{0} g\right|>e^{-a_{0}},\right. \\
& \left.\ldots,\left|\tilde{x}_{0} g R_{1, n}\right|>e^{-a_{0}}\right] \mu(d g) \\
\geq & \int_{E_{\delta}} \sum_{n=2}^{+\infty} \mathbb{E}\left[\left|\tilde{x}_{0} g R_{1, n}\right|^{-\lambda} ;\left|\tilde{x}_{0} g\right| \geq e^{\delta}>e^{-a_{0}},\left|\tilde{x}_{0} g R_{1,2}\right|>e^{-a_{0}},\right. \\
& \left.\ldots,\left|\tilde{x}_{0} g R_{1, n}\right|>e^{-a_{0}}\right] \mu(d g) \\
= & \int_{E_{\delta}}\left|\tilde{x}_{0} g\right|^{-\lambda} \sum_{m=1}^{+\infty} \mathbb{E}\left[\left|\left(\tilde{x}_{0} \cdot g\right) R_{m}\right|^{-\lambda} ;\left|\left(\tilde{x}_{0} \cdot g\right) R_{1}\right|>e^{-a_{0}-\log \left|\tilde{x}_{0} g\right|},\right. \\
& \left.\ldots,\left|\left(\tilde{x}_{0} \cdot g\right) R_{k}\right|>e^{-a_{0}-\log \left|\tilde{x}_{0} g\right|}\right] \mu(d g) \\
= & \int_{E_{\delta}}\left|\tilde{x}_{0} g\right|^{-\lambda} \Phi\left(\tilde{x}_{0} \cdot g, a_{0}+\log \left|\tilde{x}_{0} g\right|\right) \mu(d g),
\end{aligned}
$$

so that

$$
\Phi\left(\tilde{x}_{0}, a_{0}\right) \geq \int_{E_{\delta}}\left|\tilde{x}_{0} g\right|^{-\lambda} \Phi\left(\tilde{x}_{0} \cdot g, a_{0}+\delta\right) \mu(d g) .
$$

Consequently, if $\Phi\left(\tilde{x}_{0}, a_{0}\right)<+\infty$ then $\Phi\left(\tilde{x}_{0} \cdot g, a_{0}+\delta\right)<+\infty$ for $\mu$-almost all $g \in E_{\delta}$ and by iterating this argument, there thus exists a sequence $\left(g_{k}\right)_{k \geq 1}$ of elements of $E_{\delta}$ such that

$$
\forall k \geq 1, \quad \Phi\left(\tilde{x}_{0} \cdot g_{1} \ldots g_{k}, a_{0}+k \delta\right)<+\infty .
$$

By Lemma 2.1, for any $\tilde{x}, \tilde{y} \in \mathbb{X}$ and $a \in \mathbb{R}$

$$
\begin{aligned}
\Phi(\tilde{x}, a-\log c) & \leq c^{\lambda} \sum_{n=1}^{+\infty} \mathbb{E}\left[\left|R_{n}\right|^{-\lambda} ;\left|R_{1}\right|>e^{-a}, \ldots,\left|R_{n}\right|>e^{-a}\right] \\
& \leq c^{2 \lambda} \Phi(\tilde{y}, a+\log c)
\end{aligned}
$$


it follows that, by choosing $k$ sufficiently great such that $a_{0}+k \delta>a+2 \log c$, we have

$$
\Phi(\tilde{x}, a) \leq \Phi\left(\tilde{x}_{0} \cdot g_{1} \ldots g_{k}, a+2 \log c\right) \leq \Phi\left(\tilde{x}_{0} \cdot g_{1} \ldots g_{k}, a_{0}+k \delta\right)<+\infty .
$$

Step 3. For any $0 \leq k<n$, denote $L_{n, k}:=M_{n-1} \ldots M_{k}$ and $L_{n, k}=I$ otherwise. Let $c>1$ be the constant given by Lemma 2.1. For any $\tilde{x} \in \mathbb{X}$ and $a \in \mathbb{R}$, by using Lemma 2.1, we may write

$$
\begin{aligned}
\Phi_{\kappa}(\tilde{x}, a) & =\sum_{n=1}^{+\infty} \mathbb{E}\left(\left|\tilde{x} R_{n \kappa}\right|^{-\lambda} ;\left|\tilde{x} R_{\kappa}\right|>e^{-a}, \ldots,\left|\tilde{x} R_{n \kappa}\right|>e^{-a}\right) \\
& \leq c^{\lambda} \sum_{n=1}^{+\infty} \mathbb{E}\left(\left|R_{n \kappa}\right|^{-\lambda} ;\left|R_{\kappa}\right|>\frac{e^{-a}}{c}, \ldots,\left|R_{n \kappa}\right|>\frac{e^{-a}}{c}\right)
\end{aligned}
$$

so that, by duality principle and Lemma 2.1 ,

$$
\begin{aligned}
\Phi_{\kappa}(\tilde{x}, a) \leq & c^{\lambda} \sum_{n=1}^{+\infty} \mathbb{E}\left(\left|L_{n \kappa}\right|^{-\lambda} ;\left|L_{n \kappa,(n-1) \kappa}\right|>\frac{e^{-a}}{c}, \ldots,\left|L_{n \kappa}\right|>\frac{e^{-a}}{c}\right) \\
= & c^{\lambda} \sum_{n=1}^{+\infty} \mathbb{E}\left[\left|L_{n \kappa}\right|^{-\lambda} ;\left|L_{n \kappa,(n-1) \kappa}\right| \times\left|L_{(n-1) \kappa}\right|>\left|L_{(n-1) \kappa}\right| \frac{e^{-a}}{c}\right. \\
& \left.\ldots,\left|L_{n \kappa}\right|>\frac{e^{-a}}{c}\right] \\
\leq & c^{\lambda} \sum_{n=1}^{+\infty} \mathbb{E}\left(\left|L_{n \kappa}\right|^{-\lambda} ;\left|L_{n \kappa}\right|>\left|L_{(n-1) \kappa}\right| \frac{e^{-a}}{c^{2}}, \ldots,\left|L_{n \kappa}\right|>\frac{e^{-a}}{c^{2}}\right) \\
\leq & c^{2 \lambda} \sum_{n=1}^{+\infty} \mathbb{E}\left(\left|L_{n \kappa} x\right|^{-\lambda} ;\left|L_{n \kappa} x\right|>\left|L_{(n-1) \kappa} x\right| \frac{e^{-a}}{c^{4}}, \ldots,\left|L_{n \kappa} x\right|>\frac{e^{-a}}{c^{4}}\right)
\end{aligned}
$$

Consequently, setting $a_{1}:=-4 \log c$ and using the fact that the map $a \mapsto \Phi_{\kappa}(\tilde{x}, a)$ is nondecreasing for any $a \in \mathbb{R}$, one may write $\Phi_{\kappa}(\tilde{x}, a) \leq \Psi(\tilde{x})$ as long as $a<a_{1}$.

Step 4. To simplify the notation, we assume here $\kappa=1$; the proof is the same when $\kappa \geq 2$. For any $\tilde{x} \in \mathbb{X}$ and $n \geq 0$, set $X_{n}^{\prime}:=L_{n} \cdot x$ and $S_{n}^{\prime}:=\log \left|L_{n} x\right|$; the random process $\left(X_{n}^{\prime}, S_{n}^{\prime}\right)_{n \geq 0}$ is a Markov walk on $\mathbb{X} \times \mathbb{R}$ starting from $(x, 0)$ and whose transitions are governed by the ones of the Markov chain $\left(X_{n}^{\prime}\right)_{n \geq 0}$ on $\mathbb{X}$. To study the quantity $\Psi(\tilde{x})$, we follow the strategy developed in the case of one-dimensional random walks on $\mathbb{R}$ with independent increments and we thus introduce the sequence $\left(\eta_{j}\right)_{j \geq 0}$ of ladder epochs of $\left(S_{n}^{\prime}\right)_{n \geq 0}$ defined by

$$
\eta_{1}=0, \quad \eta_{j+1}=\eta_{j+1}(x):=\min \left\{n>\eta_{j}: \log \left|L_{n} x\right|>\log \left|L_{\eta_{j}} x\right|\right\}, \quad j \geq 0 .
$$


For any $\tilde{x} \in \mathbb{X}$, one may write

$$
\begin{aligned}
\Psi(\tilde{x}) & =\sum_{n=1}^{+\infty} \mathbb{E}\left(\left|L_{n} x\right|^{-\lambda} ; \exists j \geq 1 \mid n=\eta_{j}\right) \\
& =\sum_{j=1}^{+\infty} \mathbb{E}\left(\left|L_{\eta_{j}} x\right|^{-\lambda}\right) .
\end{aligned}
$$

Let $Q^{\prime}$ denote the transition kernel of the Markov walk $\left(X_{n}^{\prime}, S_{n}^{\prime}\right)_{n \geq 0}$ and $G_{Q^{\prime}}:=$ $\sum_{n=0}^{+\infty} Q^{\prime n}$ its Green kernel. The sub-process $\left(X_{\eta_{j}}^{\prime}, S_{\eta_{j}}^{\prime}\right)_{j \leq 0}$ is also a Markov chain, and its transition kernel $Q_{\eta}^{\prime}$ is given by: for any bounded Borel function $\phi: \mathbb{X} \times$ $\mathbb{R} \rightarrow \mathbb{C}$ and for any $x \in \mathbb{X}, a \in \mathbb{R}$

$$
\begin{aligned}
Q_{\eta}^{\prime} \phi(x, a)= & \mathbb{E}\left(\phi\left(X_{\eta_{1}}^{\prime}, a+S_{\eta_{1}}^{\prime}\right) \mid X_{0}^{\prime}=x\right) \\
= & \sum_{n=1}^{+\infty} \mathbb{E}\left(\phi\left(L_{n} \cdot x, a+\log \left|L_{n} x\right|\right) ; \eta_{1}=n\right) \\
= & \sum_{n=1}^{+\infty} \mathbb{E}\left[\phi\left(L_{n} \cdot x, a+\log \left|L_{n} x\right|\right) ;\right. \\
& \left.\left|L_{1} x\right| \leq 1, \ldots,\left|L_{n-1} x\right| \leq 1,\left|L_{n} x\right|>1\right] .
\end{aligned}
$$

Let $G_{\eta}^{\prime}$ denote the Green kernel associated with the process $\left(X_{\eta_{j}}^{\prime}, S_{\eta_{j}}^{\prime}\right)_{j \geq 0}$; by (4.6)

$$
\begin{aligned}
1+\Psi(\tilde{x}) & =\sum_{j=0}^{+\infty} \mathbb{E}\left(\left|L_{\eta_{j}} x\right|^{-\lambda}\right) \\
& =\sum_{j=0}^{+\infty} \int_{\mathbb{X}} \int_{\mathbb{R}} e^{-\lambda a}\left(Q_{\eta}^{\prime}\right)^{j}((x, 0), d y d a) \\
& =\int_{\mathbb{X}} \int_{\mathbb{R}} e^{-\lambda a} G_{\eta}^{\prime}((x, 0), d y d a) .
\end{aligned}
$$

The Markov walk $\left(X_{n}^{\prime}, S_{n}^{\prime}\right)_{n \geq 0}$ has been studied by many authors (see, for instance, [9, 14] or [13]). All the works are based on the fact that the transition kernel of the chain $\left(X_{n}^{\prime}\right)_{n \geq 0}$ has some "nice" spectral properties, namely its restriction to the space of Lipschitz functions on $\mathbb{X}$ is quasi-compact. In particular, it allows these authors to prove that the classical renewal theorem remains valid for this Markov walk on $\mathbb{X} \times \mathbb{R}$ as long as it is not centered, that is, $\pi_{\mu}=\lim _{n \rightarrow+\infty} \frac{1}{n} \mathbb{E}\left[\log \left|L_{n}\right|\right] \neq 0$; in this case, one may prove in particular that, for any $\tilde{x} \in \mathbb{X}$, the quantity $G_{Q^{\prime}}((x, 0), \mathbb{X} \times[0, a])$ is equivalent to $\frac{a}{\pi}$ as $a \rightarrow+\infty$ [14]. For the behaviour as $a \rightarrow+\infty$ of $G_{\eta}^{\prime}((x, 0), \mathbb{X} \times[0, a])$, the situation is way different. On one hand, it is easier since for any $j \geq 1$ the random variables $S_{\eta_{j}}^{\prime}$ are 
strictly positive, one might thus expect a similar result; on the other hand, the control of the spectrum of the transition kernel $Q_{\eta}^{\prime}$ remains unfortunately unknown in this circumstance, in particular the transition kernel $Q_{\eta}^{\prime}$ does not even act on the space of continuous functions on $\mathbb{X}$. Nevertheless, we have the following weak result with the postponed proof at the end of this subsection.

FACT 4.1. There exists $C>0$ such that for any $\tilde{x} \in \mathbb{X}$ and $a \geq 0$

$$
G_{\eta}^{\prime}((x, 0), \mathbb{X} \times[0, a])=\sum_{j=0}^{+\infty} \mathbb{P}\left(\log \left|L_{\eta_{j}} x\right| \leq a\right) \leq C a .
$$

It follows that

$$
\begin{aligned}
1+\Psi(\tilde{x}) & =\int_{\mathbb{X}} \int_{\mathbb{R}_{*}^{+}} e^{-\lambda a} G_{\eta}^{\prime}((x, 0), d y d a) \\
& \leq e^{\lambda} \sum_{a=1}^{+\infty} e^{-\lambda a} G_{\eta}^{\prime}((x, 0), \mathbb{X} \times[a-1, a]) \\
& \leq e^{\lambda} \sum_{a=1}^{+\infty} e^{-\lambda a} G_{\eta}^{\prime}((x, 0), \mathbb{X} \times[0, a]) \\
& \leq C e^{\lambda} \sum_{a=1}^{+\infty} a e^{-\lambda a}<+\infty .
\end{aligned}
$$

To complete the proof of Step 4, it remains to prove Fact 4.1. First, by definition of $E_{\delta}$, for any $j \geq 0$ and $\tilde{x} \in \mathbb{X}$, we may write $S_{\eta_{j+1}}^{\prime}-S_{\eta_{j}}^{\prime} \geq \delta 1_{E_{\delta}}\left(M_{\eta_{j}}\right)$; setting $\varepsilon_{j}:=1_{E_{\delta}}\left(M_{\eta_{j}}\right)$, this yields $S_{\eta_{j}}^{\prime} \geq \delta\left(\varepsilon_{0}+\cdots+\varepsilon_{j-1}\right)$ so that

$$
\begin{aligned}
G_{\eta}^{\prime}((x, 0), \mathbb{X} \times[0, a]) & =\sum_{j=0}^{+\infty} \mathbb{P}\left(X_{\eta_{j}}^{\prime} \in \mathbb{X}, S_{\eta_{j}}^{\prime} \in[0, a] \mid X_{0}^{\prime}=x\right) \\
& \leq \sum_{j=0}^{+\infty} \mathbb{E}\left(1_{[0, a]}\left(S_{\eta_{j}}^{\prime}\right) \mid X_{0}^{\prime}=x\right) \\
& \leq \sum_{j=0}^{+\infty} \mathbb{E}\left(1_{[0, a]}\left(\delta\left(\varepsilon_{0}+\cdots+\varepsilon_{j-1}\right)\right)\right) .
\end{aligned}
$$

To conclude, we use the fact that $\left(\varepsilon_{i}\right)_{i \geq 0}$ is a sequence of i.i.d. random variables; the elementary renewal theorem for the Bernoulli random walk process $\left[\left(\varepsilon_{0}+\cdots+\right.\right.$ $\left.\left.\varepsilon_{j-1}\right)\right]_{j \geq 0}$ implies

$$
G_{\eta}^{\prime}((x, 0), \mathbb{X} \times[0, a]) \leq \mathbb{E}\left(\sum_{j=1}^{+\infty} 1_{[0, a]}\left(\delta\left(\varepsilon_{0}+\cdots+\varepsilon_{j-1}\right)\right)\right) \preceq a .
$$


4.3. Proof of Lemma 3.2. We claim that

$$
\lim _{n \rightarrow+\infty} \widehat{\mathbb{E}}_{\tilde{x}, a}\left|\frac{1}{q_{n}^{(i)}}-\frac{1}{q_{\infty}^{(i)}}\right|=0 .
$$

By definition, the quantities $q_{n}^{(i)}$ are always less than or equal to 1. Therefore, (4.7) implies that the same property holds $\widehat{\mathbb{P}}_{\tilde{x}, a}$-almost surely for $q_{\infty}^{(i)}$. Hence,

$$
\left|q_{n}^{(i)}-q_{\infty}^{(i)}\right|=q_{n}^{(i)} q_{\infty}^{(i)}\left|\frac{1}{q_{n}^{(i)}}-\frac{1}{q_{\infty}^{(i)}}\right| \leq\left|\frac{1}{q_{n}^{(i)}}-\frac{1}{q_{\infty}^{(i)}}\right| .
$$

Using (4.7) again, we find that $\lim _{n \rightarrow+\infty} \widehat{\mathbb{E}}_{\tilde{x}, a}\left|q_{n}^{(i)}-q_{\infty}^{(i)}\right|=0$. In particular,

$$
\lim _{n \rightarrow+\infty} \widehat{\mathbb{P}}_{\tilde{x}, a}^{(i)}\left(Z_{n} \neq \tilde{\mathbf{0}}\right)=\lim _{n \rightarrow+\infty} \widehat{\mathbb{E}}_{\tilde{x}, a} q_{n}^{(i)}=\widehat{\mathbb{E}}_{\tilde{x}, a} q_{\infty}^{(i)},
$$

which is the assertion of (3.11).

Finally, it remains to verify (4.7). From (3.2) and (3.3),

$$
\begin{aligned}
\left|\frac{1}{q_{n}^{(i)}}-\frac{1}{q_{\infty}^{(i)}}\right| \leq & \left|\frac{1}{\tilde{e}_{i} R_{n} \mathbf{1}}\right| \\
& +\sum_{k=0}^{n-1}\left|\frac{\gamma_{k}}{\tilde{e}_{i} R_{k} Y_{k+1, n}}-\frac{\gamma_{k}}{\tilde{e}_{i} R_{k} Y_{k+1, \infty}}\right|+\sum_{k=n}^{+\infty}\left|\frac{\gamma_{k}}{\tilde{e}_{i} R_{k} Y_{k+1, \infty}}\right|
\end{aligned}
$$

so that

$$
\widehat{\mathbb{E}}_{\tilde{x}, a}\left|\frac{1}{q_{n}^{(i)}}-\frac{1}{q_{\infty}^{(i)}}\right| \leq I_{n}+J_{n}+K_{n}
$$

with

$$
\begin{aligned}
I_{n} & =\widehat{\mathbb{E}}_{\tilde{x}, a}\left|\frac{1}{\tilde{e}_{i} R_{n} \mathbf{1}}\right|, \\
J_{n} & =\sum_{k=0}^{n-1} \widehat{\mathbb{E}}_{\tilde{x}, a}\left|\gamma_{k} \frac{\tilde{e}_{i} R_{k}\left(Y_{k+1, n}-Y_{k+1, \infty}\right)}{\left(\tilde{e}_{i} R_{k} Y_{k+1, n}\right)\left(\tilde{e}_{i} R_{k} Y_{k+1, \infty}\right)}\right|, \\
K_{n} & =\sum_{k=n}^{+\infty} \widehat{\mathbb{E}}_{\tilde{x}, a}\left|\frac{\gamma_{k}}{\tilde{e}_{i} R_{k} Y_{k+1, \infty}}\right| .
\end{aligned}
$$

By using Lemma 2.1, it is obvious that Lemma 3.1 implies

$$
\sum_{k=0}^{+\infty} \widehat{\mathbb{E}}_{\tilde{x}, a}\left(\frac{1}{\left|R_{k}\right|}\right)<+\infty .
$$

Besides, as a direct consequence of Lemma 2.1,

$$
I_{n} \preceq \widehat{\mathbb{E}}_{\tilde{x}, a}\left(\frac{1}{\left|R_{n}\right|}\right),
$$




$$
\begin{aligned}
\left|\gamma_{k} \frac{\tilde{e}_{i} R_{k}\left(Y_{k+1, n}-Y_{k+1, \infty}\right)}{\left(\tilde{e}_{i} R_{k} Y_{k+1, n}\right)\left(\tilde{e}_{i} R_{k} Y_{k+1, \infty}\right)}\right| & \preceq \frac{\left|Y_{k+1, n}-Y_{k+1, \infty}\right|}{\left|R_{k}\right|} \preceq \frac{1}{\left|R_{k}\right|}, \\
K_{n} & \preceq \sum_{k=n}^{+\infty} \widehat{\mathbb{E}}_{\tilde{x}, a}\left(\frac{1}{\left|R_{k}\right|}\right) .
\end{aligned}
$$

Hence:

- (4.8) and (4.9) implies $I_{n} \rightarrow 0$ as $n \rightarrow \infty$.

- Since $Y_{k+1, n}(\omega) \rightarrow Y_{k+1, \infty}(\omega)$ for any $\omega \in \Omega$ (see Section 3.1), this convergence holds in particular $\hat{\mathbb{P}}_{\tilde{x}, a}$-almost surely; combining (4.8), (4.10) and the dominated convergence theorem, we obtain $J_{n} \rightarrow 0$ as $n \rightarrow+\infty$.

- By (4.8) and (4.11), the term $C_{l}$ can be made arbitrarily small by choosing $l$ sufficiently great.

Acknowledgements. We wish to thank the referees for many useful remarks and comments; their suggestions really improved the presentation of this manuscript. M. Peigné and C. Pham thank the Vietnam Institute for Advanced Studies in Mathematics (VIASM) in Ha Noi for generous hospitality and financial support during the first semester 2017; the present article was finished during this period.

\section{REFERENCES}

[1] Afanasyev, V. I. (1993). A limit theorem for a critical branching process in a random environment. Discrete Math. Appl. 5 45-58. MR1221669

[2] ATHREYA, K. B. and KARLIN, S. (1971). On branching processes with random environments. I. Extinction probabilities. Ann. Math. Stat. 42 1499-1520. MR0298780

[3] AthreyA, K. B. and KARLIN, S. (1971). Branching processes with random environments. II. Limit theorems. Ann. Math. Stat. 42 1843-1858. MR0298781

[4] BANSAYE, V. and BERESTYCKI, J. (2009). Large deviations for branching processes in random environment. Markov Process. Related Fields 15 493-524.

[5] Bougerol, Ph. and Lacroix, J. (1985). Products of Random Matrices with Applications to Schrödinger Operators. Birkhäuser, Basel.

[6] DYAKonova, E. E. (1999). The asymptotics of the probability of nonextinction of a multidimensional branching process in a random environment. Discrete Math. Appl. 9 119-136. MR1702916

[7] Dyakonova, E. E., Geiger, J. and Vatutin, V. A. (2004). On the survival probability and a functional limit theorem for branching processes in random environment. Markov Process. Related Fields 10 289-306. MR2082575

[8] Dyakonova, E. E. and Vatutin, V. A. (2017). Multitype branching processes in random environment: Survival probability for the critical case. Teor. Veroyatn. Primen. 62634 653.

[9] Furstenberg, H. and Kesten, H. (1960). Products of random matrices. Ann. Math. Stat. 31 457-469. MR0121828

[10] Geiger, J. and Kersting, G. (2000). The survival probability of a critical branching process in random environment. Teor. Veroyatn. Primen. 45 607-615. MR1967796 
[11] Geiger, J., Kersting, G. and Vatutin, V. A. (2003). Limit theorems for subcritical branching processes in random environment. Ann. Inst. Henri Poincaré Probab. Stat. 39 593-620. MR1983172

[12] Grama, I., Le Page, É. and Peigné, M. (2014). On the rate of convergence in the weak invariance principle for dependent random variables with applications to Markov chains. Colloq. Math. 134 1-55. MR3164936

[13] Grama, I., Le Page, E. and Peigné, M. (2017). Conditional limit theorems for products of random matrices. Probab. Theory Related Fields 168 601-639. MR2082575

[14] GuivarC'H, Y. and HaRdy, J. (1988). Théorèmes limites pour une classe de chaînes de Markov et applications aux difféomorphismes d'Anosov. Ann. Inst. Henri Poincaré Probab. Stat. 24 73-98. MR0937957

[15] Guivarc'H, Y., Le Page, E. and LiU, Q. (2003). Normalisation d'un processus de branchement critique dans un environnement aléatoire. C. R. Acad. Sci. Paris Sér. I Math. 337 603-608. MR2017734

[16] Guivarc'H, Y. and LiU, Q. (2001). Asymptotic properties of branching processes in a random environment. C. R. Acad. Sci. Paris Sér. I Math. 332 339-344. MR1821473

[17] Hennion, H. (1997). Limit theorems for products of positive random matrices. Ann. Probab. 25 1545-1587. MR1487428

[18] KAPLAN, N. (1974). Some results about multidimensional branching processes with random environments. Ann. Probab. 2 441-455. MR378127

[19] KozLov, M. V. (1976). On the asymptotic bahaviour of the probability of non-extinction for critical branching processes in a random environment. Theory Probab. Appl. XXI 791804. MR428492

[20] Le Page, É. (1982). Théorèmes limites pour les produits de matrices aléatoires. In Probability Measures on Groups (Oberwolfach, 1981). Lecture Notes in Math. 928 258-303. Springer, Berlin. MR0669072

[21] Pham, T. D. C. (2018). Conditioned limit theorems for products of positive random matrices. ALEA, Lat. Am. J. Probab. Math. Stat. 15 67-100.

[22] SMith, W. L. and Wilkinson, W. E. (1969). On branching processes in random environments. Ann. Math. Stat. 40 814-827. MR246380

[23] ZubKov, A. M. (1994). Inequalities for the distribution of the numbers of simultaneous events. Survey Appl. Industry Math. Ser. Probab. Stat. 1 638-666.

UNIVERSITÉ DE BRETAGNE-SUD

LMBA UMR CNRS 6205

VANNES

FRANCE

E-MAIL: emile.le-page@univ-ubs.fr
UNIVERSITÉ Fr. RABELAIS TOURS LMPT UMR CNRS 7350

TOURS

FRANCE

E-MAIL: Marc.Peigne@univ-tours.fr Da-Cam.Pham@1mpt.univ-tours.fr 